\title{
A NEW BASIS FOR THE REPRESENTATION RING OF A WEYL GROUP
}

\author{
G. LUSZTIG
}

\begin{abstract}
Let $W$ be a Weyl group. In this paper we define a new basis for the Grothendieck group of representations of $W$. This basis contains on the one hand the special representations of $W$ and on the other hand the representations of $W$ carried by the left cells of $W$. We show that the representations in the new basis have a certain bipositivity property.
\end{abstract}

\section{INTRODUCTION AND STATEMENT OF RESULTS}

0.1. Let $W$ be an irreducible Weyl group. Let $\mathcal{R}_{W}$ be the (abelian) category of finite dimensional representations of $W$ over $\mathbf{Q}$ and let $\mathcal{K}_{W}$ be the Grothendieck group of $\mathcal{R}_{W}$. Now $\mathcal{K}_{W}$ has a $\mathbf{Z}$-basis $\operatorname{Irr}_{W}$ consisting of the irreducible representations of $W$ up to isomorphism. (We often identify a representation of $W$ with its isomorphism class.)

Recall that $\operatorname{Irr}_{W}$ is partitioned into subsets called families, see [L2, §8], [L5, 4.2]; these are in 1-1 correspondence with the two-sided cells of $W$. For each family $c$ of $W$ we denote by $\mathcal{R}_{c}$ the (abelian) category of all $E \in \mathcal{R}_{W}$ which are direct sums of irreducible representations in $c$. Let $\mathcal{K}_{c}$ be the Grothendieck group of $\mathcal{R}_{c}$. It has a $\mathbf{Z}$-basis consisting of the irreducible representations in $c$. Thus we have $\mathcal{K}_{W}=\bigoplus_{c} \mathcal{K}_{c}$ where $c$ runs over the families of $W$. We now fix a family $c$ of $W$.

In [L1] we introduced a class of irreducible objects of $\mathcal{R}_{W}$ denoted by $\mathcal{S}_{W}$ (later called special representations); exactly one of these irreducible objects, denoted by $E_{c}$, is contained in $c$.

In [L4] we introduced a class of (not necessarily irreducible) objects of $\mathcal{R}_{c}$ called "cells" (later these objects were called the constructible representations). In [6] we showed that the constructible representations in $\mathcal{R}_{c}$ are precisely the representations of $W$ carried by the various left cells of $W$ contained in $c$.

In this paper we introduce a class $\mathbf{B}_{c}$ of objects of $\mathcal{R}_{c}$ which includes both $E_{c}$ and the constructible representations in $\mathcal{R}_{c}$ and which forms a $\mathbf{Z}$-basis of the group $\mathcal{K}_{c}$. The representations in $\mathbf{B}_{c}$ are called new representations. (Taking disjoint union over all families of $W$ we obtain a new $\mathbf{Z}$-basis of $\mathcal{K}_{W}$.)

0.2. Let $\Gamma$ be a finite group. As in $[\mathrm{L} 2]$ we define $M(\Gamma)$ to be the set of all pairs $(x, \rho)$ where $x \in \Gamma$ and $\rho \in \operatorname{Irr}(Z(x))$ where $Z(x)$ is the centralizer of $x$ in $\Gamma$ and $\operatorname{Irr}(Z(X))$ is the set of irreducible representations of $Z(x)$ over $\mathbf{C}$ up to isomorphism; these pairs are taken up to conjugacy by any element of $\Gamma$. Let $\mathbf{C}[M(\Gamma)]$ be the $\mathbf{C}$-vector space with basis $\{(x, \rho) ;(x, \rho) \in M(\Gamma)\}$.

Received by the editors Jne 24, 2019.

2010 Mathematics Subject Classification. Primary 20 G99.

The author was supported by NSF grant DMS-1566618. 
Let $H$ be a subgroup of $\Gamma$. For $x \in \Gamma$ let $(\Gamma / H)^{x}$ be the fixed point set of the left translation action of $x$ on $\Gamma / H$ and let $\mathbf{C}\left[(\Gamma / H)^{x}\right]$ be the $\mathbf{C}$-vector space with basis $(\Gamma / H)^{x}$. Now $Z(x)$ acts by left translation on $(\Gamma / H)^{x}$ and this induces a linear action of $Z(x)$ on $\mathbf{C}\left[(\Gamma / H)^{x}\right]$. If $\rho \in \operatorname{Irr}(Z(x))$, let $N_{H, H, x, \rho}$ be the multiplicity of $\rho$ in the $Z(x)$-module $\mathbf{C}\left[(\Gamma / H)^{x}\right]$. Let

$$
S_{H, H}=\bigoplus_{(x, \rho) \in M(\Gamma)} N_{H, H, x, \rho}(x, \rho) \in \mathbf{C}[M(\Gamma)] .
$$

More generally, let $H \subset H^{\prime}$ be subgroups of $\Gamma$ with $H$ normal in $H^{\prime}$. Then the obvious surjective map $\Gamma / H \rightarrow \Gamma / H^{\prime}$ restricts to a map $(\Gamma / H)^{x} \rightarrow\left(\Gamma / H^{\prime}\right)^{x}$ and this induces a linear map $\mathbf{C}\left[(\Gamma / H)^{x}\right] \rightarrow \mathbf{C}\left[\left(\Gamma / H^{\prime}\right)^{x}\right]$ (compatible with $Z(x)$ actions) whose image is denoted by $\mathcal{I}$. Now $\mathcal{I}$ is a $Z(x)$-submodule of $\mathbf{C}\left[\left(\Gamma / H^{\prime}\right)^{x}\right]$. If $\rho \in \operatorname{Irr}(Z(x))$, let $N_{H, H^{\prime}, x, \rho}$ be the multiplicity of $\rho$ in the $Z(x)$-module $\mathcal{I}$. Let

$$
S_{H, H^{\prime}}=\bigoplus_{(x, \rho) \in M(\Gamma)} N_{H, H^{\prime}, x, \rho}(x, \rho) \in \mathbf{C}[M(\Gamma)] .
$$

For example,

$$
\begin{gathered}
S_{\{1\},\{1\}}=\sum_{\rho \in \operatorname{Irr}(\Gamma)} \operatorname{dim} \rho(1, \rho), \\
S_{\{1\}, \Gamma=(1,1),}=\sum_{x \in \Gamma \text { up to conjugacy }}(x, 1) .
\end{gathered}
$$

0.3. As in [L5, §4] we attach to $c$ a finite group $\mathcal{G}_{c}$ and an imbedding $c \rightarrow M\left(\mathcal{G}_{c}\right)$. Let $M_{0}\left(\mathcal{G}_{c}\right)$ be the image of this imbedding. For $(x, \rho) \in M_{0}\left(\mathcal{G}_{c}\right)$ let $E_{x, \rho}$ be the corresponding (irreducible) representation in $c$. For any $\mathcal{E} \in \mathcal{R}_{c}$ we define $\underline{\mathcal{E}} \in \mathbf{C}\left[M\left(\mathcal{G}_{c}\right)\right]$ by $\underline{\mathcal{E}}=\sum_{(x, \rho) \in M_{0}\left(\mathcal{G}_{c}\right)}\left(E_{x, \rho}: \mathcal{E}\right)(x, \rho)$ where $\left(E_{x, \rho}: \mathcal{E}\right) \in \mathbf{N}$ is the multiplicity of $E_{x, \rho}$ in $\mathcal{E}$. Note that $\mathcal{E} \mapsto \underline{\mathcal{E}}$ defined an imbedding $\mathcal{K}_{c} \rightarrow \mathbf{C}\left[M\left(\mathcal{G}_{c}\right)\right]$.

As was pointed out in [L7], to any constructible representation $E$ in $\mathcal{R}_{c}$ one can attach a subgroup $H_{E}$ of $\mathcal{G}_{c}$, well defined up to conjugacy, such that $\underline{E}=S_{H_{E}, H_{E}}$; see $0.2($ a). Moreover,

(a) $E \mapsto H_{E}$

is an injective map from the set of constructible representations in $\mathcal{R}_{c}$ to the set of subgroups of $\mathcal{G}_{c}$ (up to conjugacy). Let $\mathfrak{F}_{c}$ be the set of subgroups of $\mathcal{G}_{c}$ which are conjugate to a subgroup in the image of the map (a). We have $\mathcal{G}_{c} \in \mathfrak{F}_{c}$. We say that $c$ is anomalous if $\{1\} \notin \mathfrak{F}_{c}$. If $W$ is of classical-type, then $c$ is not anomalous. If $W$ is of exceptional-type, then $c$ is anomalous in exactly the following cases:

(b) the unique $c$ with $|c|=2$ with $W$ of type $E_{7}$;

(c) the two $c$ with $|c|=2$ with $W$ of type $E_{8}$;

(d) the unique $c$ with $|c|=4$ with $W$ of type $G_{2}$;

(e) the unique $c$ with $|c|=11$ with $W$ of type $F_{4}$;

(f) the unique $c$ with $|c|=17$ with $W$ of type $E_{8}$.

Let $\hat{\mathfrak{F}}_{c}$ be the set of subgroups of $\mathcal{G}_{c}$ which are either $\{1\}$ or are in $\mathfrak{F}_{c}$. Let $\tilde{\Theta}_{c}$ be the set of all pairs $\left(H, H^{\prime}\right)$ where $H \in \hat{\mathfrak{F}}_{c}, H^{\prime} \in \hat{\mathfrak{F}}_{c}$ and $H$ is a normal subgroup of $H^{\prime}$. Now $\mathcal{G}_{c}$ acts on $\tilde{\Theta}_{c}$ by simultaneous conjugation. We now state our main result. 
Theorem 0.4. There exists a $\mathcal{G}_{c}$-stable subset $\Theta_{c}$ of $\tilde{\Theta}_{c}$ such that the following hold:

(i) For any $H \in \mathfrak{F}_{c}$ we have $(H, H) \in \Theta_{c}$.

(ii) We have $\left(1, \mathcal{G}_{c}\right) \in \Theta_{c}$.

(iii) For any $\left(H, H^{\prime}\right) \in \Theta_{c}$ there is a unique object $E_{H, H^{\prime}} \in \mathcal{R}_{c}$ such that $S_{H, H^{\prime}}=$ $\underline{E}_{H, H^{\prime}}$, see $0.2(\mathrm{a})$. Let $\mathbf{B}_{c}$ be the set of isomorphism classes of objects of $\mathcal{R}_{c}$ of the form $E_{H, H^{\prime}}$ for some $\left(H, H^{\prime}\right) \in \Theta_{c}$.

(iv) The map $\left(H, H^{\prime}\right) \mapsto E_{H, H^{\prime}}$ defines a bijection from the set of $\mathcal{G}_{c}$-orbits on $\Theta_{c}$ to $\mathbf{B}_{c}$. Moreover $\mathbf{B}_{c}$ is a $\mathbf{Z}$-basis of $\mathcal{K}_{c}$.

The representations in $\mathbf{B}_{c}$ are the new representations mentioned in 0.1 . From (i) we see that any constructible representation of $\mathcal{R}_{c}$ is in $\mathbf{B}_{c}$. From (ii) we see that the special representation $E_{c}$ is in $\mathbf{B}_{c}$.

In the case where $W$ is of type $A$ the theorem is trivial; we have $\mathcal{G}_{c}=\{1\}$ and $\mathbf{B}_{c}$ consists of the unique representation in $c$. The proof of the theorem for $W$ of type $B_{n}, C_{n}, D_{n}$ is given in $₫ 2$. The proof of the theorem for $W$ of exceptional-type is given in 33 .

0.5. In this paper we also define a canonical bijection $c \stackrel{\sim}{\rightarrow} \mathbf{B}_{c}, E \mapsto \hat{E}$ which has the property that for any $E \in c, E$ appears with multiplicity one in $\hat{E}$. For $E, E^{\prime}$ in $c$ let $E^{\prime}: \hat{E}$ be the multiplicity of $E^{\prime}$ in $\hat{E}$. Property (i) below will be proved in a sequel to this paper. (For $W$ of exceptional-type (i) is easily deduced from the formulas in $3.2[3.8$, )

(i) The matrix $\left(E^{\prime}: \hat{E}\right)$ indexed by $c \times c$ is upper triangular unipotent for a suitable partial order on $c$.

0.6. In the setup of 0.2 we define (following $[\mathrm{L2}, \S 4]$ ) a pairing $\{\}:, M(\Gamma) \times M(\Gamma) \rightarrow$ C by

$$
\begin{aligned}
& \left\{(x, \rho),\left(x^{\prime}, \rho^{\prime}\right)\right\} \\
& =|Z(x)|^{-1}\left|Z\left(x^{\prime}\right)\right|^{-1} \sum_{g \in \Gamma ; x g x^{\prime} g^{-1}=g x^{\prime} g^{-1} x} \overline{\operatorname{tr}\left(g^{-1} x g, \rho^{\prime}\right)} \operatorname{tr}\left(g x^{\prime} g^{-1}, \rho\right),
\end{aligned}
$$

where ${ }^{-}$is complex conjugation. We define the non-abelian Fourier transform $A$ : $\mathbf{C}[M(\Gamma)] \rightarrow \mathbf{C}[M(\Gamma)]$ as the $\mathbf{C}$-linear map such that

$$
A(x, \rho)=\sum_{\left(x^{\prime}, \rho^{\prime}\right) \in M(\Gamma)}\left\{(x, \rho),\left(x^{\prime}, \rho^{\prime}\right)\right\}\left(x^{\prime}, \rho^{\prime}\right)
$$

for any $(x, \rho) \in M(\Gamma)$. According to $\left[\mathrm{L} 2\right.$, we have $A^{2}=1$. Let $M(\Gamma)_{\geq 0}$ be the set of elements

$$
\sum_{(x, \rho) \in M(\Gamma)} c_{x, \rho}(x, \rho) \in \mathbf{C}[M(\Gamma)]
$$

such that $c_{x, \rho} \in \mathbf{R}_{\geq 0}$ for any $(x, \rho) \in M(\Gamma)$.

An element $f \in \mathbf{C}[M(\Gamma)]$ is said to be bipositive if $f \in M(\Gamma)_{\geq 0}$ and $A(f) \in$ $M(\Gamma)_{\geq 0}$. We have the following result.

Theorem 0.7. Let $H \subset H^{\prime}$ be subgroups of $\Gamma$ with $H$ normal in $H^{\prime}$. Then $S_{H, H^{\prime}} \in$ $\mathbf{C}[M(\Gamma)]$ is bipositive. Hence (by 0.4$)$, if $\Gamma=\mathcal{G}_{c}$ and $\mathcal{E}$ is a new representation in $\mathcal{R}_{c}$, then $\underline{\mathcal{E}} \in \mathbf{C}[M(\Gamma)]$ is bipositive.

The proof is given in $\$$. 
0.8. In a sequel to this paper we will extend the results of the paper by constructing a new basis for $\mathbf{C}\left[M\left(\mathcal{G}_{c}\right)\right]$ consisting of bipositive elements; this provides a new $\mathbf{Z}$ basis for the Grothendieck group of unipotent representations of a split Chevalley group over a finite field.

0.9. Notation. For $a \leq b$ in $\mathbf{N}$ we write $[a, b]=\{z \in \mathbf{N} ; a \leq z \leq b\}$. We set $[1,0]=\emptyset$. For a finite set $Y$ we write $|Y|$ for the cardinal of $Y$. For $a, b$ in $\mathbf{Z}$ we write $a={ }_{2} b$ if $a=b \bmod 2$ and $a \neq{ }_{2} b$ if $a \neq b \bmod 2$. We write $\mathbf{Z} / 2 \mathbf{Z}=\mathbf{F}_{2}$.

\section{The SET $S_{D}$}

1.1. Let $D \in \mathbf{N}$. A subset $I$ of $[1, D]$ is said to be an interval if $I=[a, b]$ for some $a \leq b$ in $[1, D]$. Let $\mathcal{I}_{D}$ be the set of intervals of $[1, D]$. For $I=[a, b], I^{\prime}=\left[a^{\prime}, b^{\prime}\right]$ in $\mathcal{I}_{D}$ we write $I \prec I^{\prime}$ whenever $a^{\prime}<a \leq b<b^{\prime}$. We say that $I, I^{\prime}$ are non-touching (and we write $I \boldsymbol{\wedge} I^{\prime}$ ) if $a^{\prime}-b \geq 2$ or $a-b^{\prime} \geq 2$. Let $\mathcal{I}_{D}^{1}=\left\{I \in \mathcal{I}_{D} ;|I|=\right.$ odd $\}$. Let $R_{D}^{1}$ be the set whose elements are the subsets of $\mathcal{I}_{D}^{1}$. Let $\emptyset \in R_{D}^{1}$ be the empty subset of $\mathcal{I}_{D}^{1}$.

When $D \geq 2$ and $i \in[1, D]$ we define an (injective) map $\xi_{i}: \mathcal{I}_{D-2} \rightarrow \mathcal{I}_{D}$ as follows:

$$
\xi_{i}\left(\left[a^{\prime}, b^{\prime}\right]\right)=\left[a^{\prime}+2, b^{\prime}+2\right] \text { if } i \leq a^{\prime}, \quad \xi_{i}\left(\left[a^{\prime}, b^{\prime}\right]\right)=\left[a^{\prime}, b^{\prime}\right] \text { if } i \geq b^{\prime}+2,
$$

$$
\xi_{i}\left(\left[a^{\prime}, b^{\prime}\right]\right)=\left[a^{\prime}, b^{\prime}+2\right] \text { if } a^{\prime}<i<b^{\prime}+2 .
$$

We have $\xi_{i}\left(\mathcal{I}_{D-2}^{1}\right) \subset \mathcal{I}_{D}^{1}$. We define $t_{i}: R_{D-2}^{1} \rightarrow R_{D}^{1}$ by $B^{\prime} \mapsto\left\{\xi_{i}\left(I^{\prime}\right) ; I^{\prime} \in B^{\prime}\right\} \sqcup\{i\}$. We have $\left|t_{i}\left(B^{\prime}\right)\right|=\left|B^{\prime}\right|+1$.

1.2. We define a subset $S_{D}$ of $R_{D}^{1}$ by induction on $D$ as follows. When $D \in\{0,1\}$, $S_{D}$ consists of a single element, namely $\emptyset \in R_{D}^{1}$. When $D \geq 2$ we say that $B \in R_{D}^{1}$ is in $S_{D}$ if either $B=\emptyset$ or if

(i) there exists $i \in[1, D]$ (if $D$ is even) or $i \in[1, D-1]$ (if $D$ is odd) and $B^{\prime} \in S_{D-2}$ such that $B=t_{i}\left(B^{\prime}\right)$.

If $D$ is odd, we have $S_{D}=S_{D-1}$ (use induction on $D$ ).

Until the end of 1.8 we assume that $D$ is even.

1.3. The set $S_{D}^{\prime}$. Let $B \in R_{D}^{1}$. We consider the following properties $\left(P_{0}\right),\left(P_{1}\right)$ that $B$ may or may not have.

$\left(P_{0}\right)$ If $I \in B, \tilde{I} \in B$, then either $I=\tilde{I}$, or $I \mathbf{\Lambda} \tilde{I}$, or $I \prec \tilde{I}$, or $\tilde{I} \prec I$.

$\left(P_{1}\right)$ If $[a, b] \in B$ and $c \in \mathbf{N}$ satisfies $a<c<b, a-c={ }_{2} 1$ (hence $b-c={ }_{2} 1$ ), then there exists $\left[a_{1}, b_{1}\right] \in B$ such that $a<a_{1} \leq c \leq b_{1}<b$.

From the definitions we see that if $D \geq 2, i \in[1, D], B^{\prime} \in R_{D-2}^{1}$ and $B=$ $t_{i}\left(B^{\prime}\right) \in R_{D}^{1}$, the following holds:

(a) $B^{\prime}$ satisfies $\left(P_{0}\right)$ if and only if $B$ satisfies $\left(P_{0}\right) ; B^{\prime}$ satisfies $\left(P_{1}\right)$ if and only if $B$ satisfies $\left(P_{1}\right)$.

Let $S_{D}^{\prime}$ be the set of all $B \in R_{D}^{1}$ which satisfy $\left(P_{0}\right),\left(P_{1}\right)$. In the setup of (a) we have the following consequence of (a):

(b) We have $B^{\prime} \in S_{D-2}^{\prime}$ if and only if $B \in S_{D}^{\prime}$.

We show:

(c) $S_{D}=S_{D}^{\prime}$.

We argue by induction on $D$. If $D=0, S_{D}^{\prime}$ consists of the empty set hence (c) holds in this case. Assume now that $D \geq 2$. Let $B \in S_{D}$. We show that $B \in S_{D}^{\prime}$. If $B=\emptyset$ this is clear. If $B \neq \emptyset$, then $B=t_{i}\left(B^{\prime}\right)$ for some $i, B^{\prime} \in S_{D-2}$. By the 
induction hypothesis we have $B^{\prime} \in S_{D-2}^{\prime}$. By (b) we have $B \in S_{D}^{\prime}$. We see that $B \in S_{D} \Longrightarrow B \in S_{D}^{\prime}$. Conversely, let $B \in S_{D}^{\prime}$. We show that $B \in S_{D}$. If $B=\emptyset$ this is obvious. Thus we can assume that $B \neq \emptyset$. Let $[a, b] \in B$ be such that $b-a$ is minimum. If $a<z<b, z={ }_{2} a+1$, then by $\left(P_{1}\right)$ we have $z \in\left[a^{\prime}, b^{\prime}\right]$ with $\left[a^{\prime}, b^{\prime}\right] \in B$, $b^{\prime}-a^{\prime}<b-a$, contradicting the minimality of $b-a$. We see that no $z$ as above exists. Thus, $[a, b]=\{i\}$ for some $i \in[1, D]$. Using $\left(P_{0}\right)$ and $\{i\} \in B$, we see that $B$ does not contain any interval of the form $[a, i]$ with $a<i$, or $[i, b]$ with $i<b$, or $[a, i-1]$ with $a<i$ or $[i+1, b]$ with $i<b$; hence any interval of $B$ other than $\{i\}$ is of the form $\xi_{i}\left[a^{\prime}, b^{\prime}\right]$ where $\left[a^{\prime}, b^{\prime}\right] \in \mathcal{I}_{D-2}^{1}$. Thus we have $B=t_{i}\left(B^{\prime}\right)$ for some $B^{\prime} \in S_{D-2}$. From (b) we see that $B^{\prime} \in S_{D-2}^{\prime}$. Using the induction hypothesis we deduce that $B^{\prime} \in S_{D-2}$. By the definition of $S_{D}$, we have $B \in S_{D}$. This completes the proof of $(\mathrm{c})$.

The following result has already been proved as a part of the proof of (c).

(d) Assume that $D \geq 2, i \in[1, D]$. Let $B \in S_{D}$ be such that $\{i\} \in B$. Then there exists $B^{\prime} \in S_{D-2}$ such that $B=t_{i}\left(B^{\prime}\right)$.

1.4. For $B \in S_{D}, j \in[1, D]$ we set $B_{j}=\{I \in B ; j \in I\}$. From the definitions we deduce:

(a) Assume that $D \geq 2, i \in[1, D]$ and that $B^{\prime} \in S_{D-2}, B=t_{i}\left(B^{\prime}\right) \in S_{D}$. Then for $r \in[1, D-2]$ we have:

$\left.\mid B_{r}^{\prime}\right)|=| B_{r} \mid$ if $r \leq i-2,\left|B_{r}^{\prime}\right|=\left|B_{r+2}\right|$ if $r \geq i$,

$\left|B_{i-1}\right|=\left|B_{i+1}\right|=\left|B_{i-1}^{\prime}\right|,\left|B_{i}\right|=\left|B_{i-1}^{\prime}\right|+1$ if $1<i<D$,

$\left|B_{i-1}\right|=0$ if $i=D,\left|B_{i+1}\right|=0$ if $i=1$.

1.5. Let $B \in S_{D}, B \neq \emptyset$. In this case we must have $\{j\} \in B$ for some $j \in[1, D]$; we assume that $j$ is as small as possible (then it is uniquely determined). As in the proof of 1.3 (c) we have $B=t_{j}\left(B^{\prime}\right)$ where $B^{\prime} \in S_{D-2}$. Let $i$ be the smallest number in $\bigcup_{I \in B} I$. We have $i \leq j$. We show:

(a) For any $h \in[i, j]$, we have $[h, \tilde{h}] \in B$ for a unique $\tilde{h} \in[h, D]$; moreover we have $j \leq \tilde{h}$.

We argue by induction on $D$. When $D=0$ the result is obvious. We now assume that $D \geq 2$. Assume first that $i=j$. By $\left(P_{0}\right),\{j\} \in B$ implies that we cannot have $[j, b] \in B$ with $j<b$; thus (a) holds in this case. In particular, (a) holds when $D=2$ (in this case we have $i=j$ ). We now assume that $D \geq 4$. We can assume that $i<j$. We have $[i, b] \in B$ for some $b>i$ hence $|B| \geq 2$ so that $\left|B^{\prime}\right| \geq 1$ and $B^{\prime} \neq \emptyset$. Then $i^{\prime}, j^{\prime}$ are defined in terms of $B^{\prime}$ in the same way as $i, j$ are defined in terms of $B$. From $\left(P_{1}\right)$ we see that there exists $j_{1}$ such that $i<j_{1}<b$ such that $\left\{j_{1}\right\} \in B$. By the minimality of $j$ we must have $j \leq j_{1}$. Thus we have $i<j<b$. We have $\left.[i, b]=\xi_{j}[i, b-2]\right]$ hence $[i, b-2] \in B^{\prime}$. This implies that $i^{\prime} \leq i$. We have $\left[i^{\prime}, c\right] \in B^{\prime}$ for some $c \in\left[i^{\prime}, D-2\right], c={ }_{2} i^{\prime}$; hence $\left[i^{\prime}, c^{\prime}\right] \in B$ for some $c^{\prime} \geq i^{\prime}$ so that $i^{\prime} \geq i$. Thus we have $i^{\prime}=i$. By the induction hypothesis, the following holds:

(b) For any $r \in\left[i, j^{\prime}\right]$, we have $\left[r, r_{1}\right] \in B^{\prime}$ for a unique $r_{1}$; moreover $j^{\prime} \leq r_{1}$.

If $j^{\prime} \leq j-2$, then $\left\{j^{\prime}\right\}=\xi_{j}\left(\left\{j^{\prime}\right\}\right) \in B$. Hence $j^{\prime} \geq j$ by the minimality of $j$; this is a contradiction. Thus we have $j^{\prime} \geq j-1$.

Let $r \in[i, j-1]$. Then we have also $r \in\left[i, j^{\prime}\right]$ hence $r_{1}$ is defined as in (b). We have $\left[r, r_{1}\right] \in B^{\prime}$ hence $\left[r, r_{1}+2\right] \in B$ (we use that $r<j \leq j^{\prime}+1 \leq r_{1}+1<r_{1}+2$ ); we have $j<r_{1}+2$. Assume now that $\left[r, r_{2}\right] \in B$ with $r \leq r_{2}$. Then $r<r_{2}$ (by the minimality of $j$ ). If $j=r_{2}$ or $j=r_{2}+1$, then applying $\left(P_{0}\right)$ to $\{j\},\left[r, r_{2}\right]$ gives a contradiction. Thus we must have either $r<j<r_{2}$ or $j>r_{2}+1$. If $j>r_{2}+1$, 
then $\left[r, r_{2}\right] \in B^{\prime}$ hence by (b), $r_{2}=r_{1}$, hence $j>r_{1}+1$ contradicting $j<r_{1}+2$. Thus we have $r<j<r_{2}$, so that $\left[r, r_{2}-2\right] \in B^{\prime}$ hence by (b), $r_{2}-2=r_{1}$. Thus we have $r<j<r_{2}$ so that $\left[r, r_{2}-2\right] \in B^{\prime}$ hence by (b), $r_{2}-2=r_{1}$.

Next we assume that $r=j$. In this case we have $\{r\} \in B$. Moreover, if $\left[r, r^{\prime}\right] \in B$ with $r \leq r^{\prime} \leq D$, then we cannot have $r<r^{\prime}$ (if $r<r^{\prime}$, then applying $\left(P_{0}\right)$ to $\{r\},\left[r, r^{\prime}\right]$ gives a contradiction). This proves (a).

We show:

(c) Assume that $j<D$ and that $i \leq h<j$. Then $\tilde{h}$ in (a) satisfies $\tilde{h}>j$.

Assume that $\tilde{h}=j$, so that $[h, j] \in B$. Since $h<j$, applying $\left(P_{0}\right)$ to $\{j\},[h, j]$ gives a contradiction. This proves (c).

(d) Assume that $j<D$ and that $r \in[j+1, D]$. We have $[j+1, r] \notin B$.

Assume that $[j+1, r] \in B$. Applying $\left(P_{0}\right)$ to $\{j\},[j+1, r]$ gives a contradiction. This proves $(\mathrm{d})$.

We show:

(e) For $h \in[i, j]$ we have $\left|B_{h}\right|=h-i+1$. If $j<D$ we have $\left|B_{j+1}\right|=j-i$.

Let $h \in[i, j]$. Then for any $h^{\prime} \in[i, h], B_{h}$ contains $\left[h^{\prime}, \tilde{h}^{\prime}\right]$ (since $h \leq \tilde{h}^{\prime}$ ); see (a). Conversely, assume that $[a, b] \in B_{h}$. We have $a \leq h$. By the definition of $i$ we have $i \leq a$. By the uniqueness statement in (a) we have $b=\tilde{a}$ so that $[a, b]$ is one of the $h-i+1$ intervals $\left[h^{\prime}, \tilde{h}^{\prime}\right]$ above. This proves the first assertion of (e). Assume now that $j<D$. If $h^{\prime} \in[i, j], h^{\prime}<j$, then $\left[h^{\prime}, \tilde{h}^{\prime}\right] \in B_{j+1}$, by (c). Conversely, assume that $[a, b] \in B_{j+1}$. We have $a \leq j+1$ and by (d) we have $a \neq j+1$ so that $a \leq j$. If $a=j$, then by the uniqueness in (a) we have $b=j$ which contradicts $j+1 \in[a, b]$. Thus we have $a \leq j-1$. We see that $[a, b]$ is one of the $j-i$ intervals $\left[h^{\prime}, \tilde{h}^{\prime}\right]$ with $h^{\prime} \in[i, j], h^{\prime}<j$. This proves (e).

1.6. For $B \in S_{D}, j \in[1, D]$, we set

$$
\epsilon_{j}(B)=\left|B_{j}\right|\left(\left|B_{j}\right|+1\right) / 2 \in \mathbf{F}_{2} .
$$

We have $\epsilon_{j}(B)=1$ if $\left|B_{j}\right| \in(4 \mathbf{Z}+1) \cup(4 \mathbf{Z}+2), \epsilon_{j}(B)=0$ if $\left|B_{j}\right| \in(4 \mathbf{Z}+3) \cup(4 \mathbf{Z})$.

Assume now that $B \neq \emptyset$. Let $i \leq j$ in $[1, D]$ be as in 1.5. From 1.5)(e) we deduce:

(a) We have $\left(\left|B_{i}\right|,\left|B_{i+1}\right|, \ldots,\left|B_{j}\right|\right)=(1,2,3, \ldots, j-i, j-i+1)$. If $j<D$, we have $\left|B_{j+1}\right|=j-i$.

From (a) we deduce:

(b)

$$
\begin{aligned}
& \left(\epsilon_{i}(B), e_{i+1}(B), \ldots, \epsilon_{j}(B)\right) \\
& =(1 \times 2) / 2,(2 \times 3) / 2,(3 \times 4) / 2, \ldots,(j-i)(j-i+1) / 2,(j-i+1)(j-i+2) / 2) ; \\
& \quad \text { (c) if } j<D, \text { then } \epsilon_{j+1}(B)=(j-i)(j-i+1) / 2 . \\
& \quad \text { For future reference we note: } \\
& \quad \text { (d) If } c \in \mathbf{Z} \text {, then } c(c+1) / 2 \neq_{2}(c+2)(c+3) / 2 . \\
& \quad \text { (e) If } c \in 2 \mathbf{Z} \text {, then } c(c+1) / 2 \neq_{2}(c+1)(c+2) / 2 .
\end{aligned}
$$

1.7. Let $B \in S_{D}, \tilde{B} \in S_{D}$ be such that $B \neq \emptyset, \tilde{B} \neq \emptyset$ and $\epsilon_{h}(B)=\epsilon_{h}(\tilde{B})$ for any $h \in[1, D]$. We show:

(a) We can find $z \in[1, D]$ such that $\{z\} \in B,\{z\} \in \tilde{B}$.

We associate $i \leq j$ to $B$ as in 1.5 let $\tilde{i} \leq \tilde{j}$ be the analogous number for $\tilde{B}$. Assume first that $j<\tilde{j}$ (so that $j<D$ ) and $i<\tilde{i}$. From 1.6 for $B$ we have $\epsilon_{i}(B)=(1 \times 2) / 2=1$. Since $i<\tilde{i}$ we have $\epsilon_{i}(\tilde{B})=0$. Hence $1={ }_{2} 0$, a contradiction. Thus we must have $i \geq \tilde{i}$. 
Next we asssume that $j<\tilde{j}$ (so that $j<D$ ) and $\tilde{i}<i$. From 1.6 for $\tilde{B}$ we have $\epsilon_{\tilde{i}}(\tilde{B})=(1 \times 2) / 2$; moreover $\epsilon_{\tilde{i}}(B)=0$. Hence $1={ }_{2} 0$, a contradiction. Thus when $j<\tilde{j}$ we must have $i=\tilde{i}$. From 1.6(c) for $B$ we have $e_{j+1}(B)=(j-i)(j-i+1) / 2$ and from 1.6(b) for $\tilde{B}$ we have $e_{j+1}(\tilde{B})=(j-i+2)(j-i+3) / 2$. It follows that

$$
(j-i)(j-i+1) / 2)=_{2}(j-i+2)(j-i+3) / 2,
$$

contradicting [1.6(d). We see that $j<\tilde{j}$ leads to a contradiction. Similarly, $\tilde{j}<j$ leads to a contradiction. Thus we must have $j=\tilde{j}$, so that (a) holds with $z=j=\tilde{j}$. This completes the proof of (a).

1.8. Let $B \in S_{D}, \tilde{B} \in S_{D}$.

(a) Assume that $\tilde{B}=\emptyset$ and that $\epsilon_{h}(B)=\epsilon_{h}(\tilde{B})$ for any $h \in[1, D]$. Then $\tilde{B}=B$.

The proof is similar to that of 1.7(a). Assume that $B \neq \emptyset$. Let $i \leq j$ be attached to $B$ as in 1.5 .

Using 1.6 we see that $e_{i}(B)=(1 \times 2) / 2$. On the other hand we have $e_{i}(\tilde{B})=0$. We get $1={ }_{2} 0$, a contradiction. This proves (a).

1.9. We no longer assume that $D$ is even. Let $V$ be the $\mathbf{F}_{2}$-vector space consisting of all functions $[1, D] \rightarrow \mathbf{F}_{2}$. For any subset $I$ of $[1, D]$ let $e_{I} \in V$ be the function whose value at $i$ is 1 if $i \in I$ and is 0 if $i \notin I$. For $i \in[1, D]$ we set $e_{i}=e_{\{i\}}$. Now $\left\{e_{i} ; i \in[1, D]\right\}$ is a basis of $V$. We define a symplectic form $():, V \times V \rightarrow \mathbf{F}_{2}$ by $\left(e_{i}, e_{j}\right)=1$ if $i-j= \pm 1,\left(e_{i}, e_{j}\right)=0$ if $i-j \neq \pm 1$. This symplectic form is non-degenerate if $D$ is even while if $D$ is odd it has a one dimensional radical spanned by $e_{1}+e_{3}+e_{5}+\cdots+e_{D}$.

For any subset $Z$ of $V$ we set $Z^{\perp}=\{x \in V ;(x, z)=0 \quad \forall z \in Z\}$.

When $D \geq 2$ we denote by $V^{\prime}$ the $\mathbf{F}_{2}$-vector space consisting of all functions $[1, D-2] \rightarrow \mathbf{F}_{2}$. For any $I^{\prime} \subset[1, D-2]$ let $e_{I^{\prime}}^{\prime} \in V^{\prime}$ be the function whose value at $i$ is 1 if $i \in I^{\prime}$ and is 0 if $i \notin I^{\prime}$. For $i \in[1, D-2]$ we set $e_{i}^{\prime}=e_{\{i\}}^{\prime}$. Now $\left\{e_{i}^{\prime} ; i \in[1, D-2]\right\}$ is a basis of $V^{\prime}$. We define a symplectic form $(,)^{\prime}: V^{\prime} \times V^{\prime} \rightarrow \mathbf{F}_{2}$ by $\left(e_{i}^{\prime}, e_{j}^{\prime}\right)=1$ if $i-j= \pm 1,\left(e_{i}^{\prime}, e_{j}^{\prime}\right)=0$ if $i-j \neq \pm 1$.

When $D \geq 2$, for any $i \in[1, D]$ there is a unique linear map $T_{i}: V^{\prime} \rightarrow V$ such that the sequence $T_{i}\left(e_{1}^{\prime}\right), T_{i}\left(e_{2}^{\prime}\right), \ldots, T_{i}\left(e_{D-2}^{\prime}\right)$ is:

$$
\begin{aligned}
& e_{1}, e_{2}, \ldots, e_{i-2}, e_{i-1}+e_{i}+e_{i+1}, e_{i+2}, e_{i+3}, \ldots, e_{D}(\text { if } 1<i<D), \\
& e_{3}, e_{4}, \ldots, e_{D}(\text { if } i=1), \\
& e_{1}, e_{2}, \ldots, e_{D-2}(\text { if } i=D) .
\end{aligned}
$$

Note that $T_{i}$ is injective and $(x, y)^{\prime}=\left(T_{i}(x), T_{i}(y)\right)$ for any $x, y$ in $V^{\prime}$. For any $I^{\prime} \in \mathcal{I}_{D-2}^{1}$ we have $T_{i}\left(e_{I^{\prime}}^{\prime}\right)=e_{\xi_{i}\left(I^{\prime}\right)}$. Let $V_{i}$ be the image of $T_{i}: V^{\prime} \rightarrow V$. From the definitions we deduce:

(a) We have $e_{i}^{\perp}=V_{i} \oplus \mathbf{F}_{2} e_{i}$.

We now assume that $D$ is even. For $j \in[1, D-2]$ let $\epsilon_{j}^{\prime}: S_{D-2} \rightarrow \mathbf{F}_{2}$ be the analogue of $\epsilon_{i}: S_{D} \rightarrow \mathbf{F}_{2}$ when $D$ is replaced by $D-2$.

For $B \in S_{D}$, we define $\epsilon(B) \in V$ by $i \mapsto \epsilon_{i}(B)$. For $B^{\prime} \in S_{D-2}$ we define $\epsilon^{\prime}\left(B^{\prime}\right) \in V^{\prime}$ by $j \mapsto \epsilon_{j}^{\prime}\left(B^{\prime}\right)$. We show:

(b) Assume that $D \geq 2, i \in[1, D]$. Let $B^{\prime} \in S_{D-2}, B=t_{i}\left(B^{\prime}\right) \in S_{D}$. Then $\epsilon(B)=T_{i}\left(\epsilon^{\prime}\left(B^{\prime}\right)\right)+$ ce $e_{i}$ for some $c \in \mathbf{F}_{2}$.

An equivalent statement is: for any $j \in[1, D]-\{i\}$ we have $\epsilon_{j}(B)=\epsilon_{j^{\prime}}^{\prime}\left(B^{\prime}\right)$ if $j^{\prime} \in[1, D-2]$ is such that $j \in \xi_{i}\left(\left\{j^{\prime}\right\}\right)$; and $\epsilon_{j}(B)=0$ if no such $j^{\prime}$ exists. It is enough to show:

$$
\left|B_{h}^{\prime}\right|=\left|B_{h}\right| \text { if } h \in[1, i-2],
$$


$\left|B_{h-2}^{\prime}\right|=\left|B_{h}\right|$ if $h \in[i+2, D]$,

$\left|B_{i-1}\right|=\left|B_{i+1}\right|=\left|B_{i-1}^{\prime}\right|$ if $1<i<D$,

$\left|B_{i-1}\right| \in\{0,-1\}$ (hence $\epsilon_{i-1}(B)=0$ ) if $i=D$,

$\left|B_{i+1}\right| \in\{0,-1\}$ (hence $\epsilon_{i+1}(B)=0$ ) if $i=1$.

This follows from 1.4(a).

For $B \in S_{D}$ let $\langle B\rangle$ be the subspace of $V$ generated by $\left\{e_{I} ; I \in B\right\}$. For $B^{\prime} \in S_{D-2}$ let $\left\langle B^{\prime}\right\rangle$ be the subspace of $V^{\prime}$ generated by $\left\{e_{I^{\prime}}^{\prime} ; I^{\prime} \in B^{\prime}\right\}$. We show:

(c) Let $B \in S_{D}$. We have $\epsilon(B) \in\langle B\rangle$. If $D \geq 2, i \in[1, D], B^{\prime} \in S_{D-2}, B=$ $t_{i}\left(B^{\prime}\right) \in S_{D}$, then $\langle B\rangle=T_{i}\left(\left\langle B^{\prime}\right\rangle\right) \oplus \mathbf{F}_{2} e_{i}$.

To prove the first assertion of (c) we argue by induction on $D$. For $D=0$ there is nothing to prove. Assume that $D \geq 2$. Let $i, B^{\prime}$ be as in (b). By the induction hypothesis we have $\epsilon^{\prime}\left(B^{\prime}\right) \in\left\langle B^{\prime}\right\rangle \subset V^{\prime}$. Using (b) we see that it is enough to show that $T_{i}\left(\left\langle B^{\prime}\right\rangle\right) \subset\langle B\rangle$. (Since $\{i\} \in B$, we have $e_{i} \in\langle B\rangle$.) Using the equality $T_{i}\left(e_{I^{\prime}}^{\prime}\right)=e_{\xi_{i}\left(I^{\prime}\right)}$ for any $I^{\prime} \in B^{\prime}$ it remains to note that $\xi_{i}\left(I^{\prime}\right) \in B$ for $I^{\prime} \in B^{\prime}$. This proves the first assertion of (c). The same proof shows the second assertion of (c).

1.10. Let $B \in S_{D}, \tilde{B} \in S_{D}$. We show:

(a) If $\epsilon(B)=\epsilon(\tilde{B})$, then $B=\tilde{B}$.

We argue by induction on $D$. If $D=0$, there is nothing to prove. Assume that $D \geq 2$. If $\tilde{B}=\emptyset$, (a) follows from 1.8 (a). Similarly, (a) holds if $B=\emptyset$. Thus, we can assume that $B \neq \emptyset, \tilde{B} \neq \emptyset$. By 1.7 (a) we can find $i \in[1, D]$ such that $\{i\} \in B$, $\{i\} \in \tilde{B}$. By 1.3 (d) we then have $B=t_{i}\left(B^{\prime}\right), \tilde{B}=t_{i}\left(\tilde{B}^{\prime}\right)$ with $B^{\prime} \in S_{D-2}, \tilde{B}^{\prime} \in$ $S_{D-2}$. Using our assumption and 1.9 (b) we see that $T_{i}\left(\epsilon^{\prime}\left(B^{\prime}\right)\right)=T_{i}\left(\epsilon^{\prime}\left(\tilde{B}^{\prime}\right)\right)+c e_{i}$ for some $c \in \mathbf{F}_{2}$. Using [1.9(a) we see that $c=0$ so that $T_{i}\left(\epsilon^{\prime}\left(B^{\prime}\right)\right)=T_{i}\left(\epsilon^{\prime}\left(\tilde{B}^{\prime}\right)\right)$. Since $T_{i}$ is injective, we deduce $\epsilon^{\prime}\left(B^{\prime}\right)=\epsilon^{\prime}\left(\tilde{B}^{\prime}\right)$. By the induction hypothesis we have $B^{\prime}=\tilde{B}^{\prime}$ hence $B=\tilde{B}$. This proves (a).

1.11. Any $x \in V$ can be written uniquely in the form

$$
x=e_{\left[a_{1}, b_{1}\right]}+e_{\left[a_{2}, b_{2}\right]}+\cdots+e_{\left[a_{r}, b_{r}\right]},
$$

where $\left[a_{r}, b_{r}\right] \in \mathcal{I}_{D}$ are such that any two of them are non-touching and $r \geq 0$, $1 \leq a_{1} \leq b_{1}<a_{1} \leq b_{2}<\cdots<a_{r} \leq b_{r} \leq D$. Following [L3, 3.3] we set

$$
u(v)=\left|\left\{s \in[1, r] ; a_{s}={ }_{2} 0, b_{s}={ }_{2} 1\right\}\right|-\left|\left\{s \in[1, r] ; a_{s}={ }_{2} 1, b_{s}={ }_{2} 0\right\}\right| \in \mathbf{Z} .
$$

This defines a function $u: V \rightarrow \mathbf{Z}$. When $D \geq 2$ we denote by $u^{\prime}: V^{\prime} \rightarrow \mathbf{Z}$ the analogous function with $D$ replaced by $D-2$. We show:

(b) Assume that $D \geq 2, i \in[1, D]$. Let $v^{\prime} \in V^{\prime}$ and let $v=T_{i}\left(v^{\prime}\right)+c e_{i} \in V$ where $c \in \mathbf{F}_{2}$. We have $u(v)=u^{\prime}\left(v^{\prime}\right)$.

We write $v^{\prime}=e_{\left[a_{1}^{\prime}, b_{1}^{\prime}\right]}^{\prime}+e_{\left[a_{2}^{\prime}, b_{2}^{\prime}\right]}^{\prime}+\cdots+e_{\left[a_{r}^{\prime}, b_{r}^{\prime}\right]}^{\prime}$ where $r \geq 0,\left[a_{s}^{\prime}, b_{s}^{\prime}\right] \in \mathcal{I}_{D-2}$ for all $s$ and any two of $\left[a_{s}^{\prime}, b_{s}^{\prime}\right]$ are non-touching. For each $s$, we have $T_{i}\left(e_{\left[a_{s}^{\prime}, b_{s}^{\prime}\right]}^{\prime}\right)=e_{\left[a_{s}, b_{s}\right]}$ where $\left[a_{s}, b_{s}\right]=\xi_{i}\left[a_{s}^{\prime}, b_{s}^{\prime}\right]$ so that $a_{s}={ }_{2} a_{s}^{\prime}, b_{s}={ }_{2} b_{s}^{\prime}$ and the various $\left[a_{s}, b_{s}\right]$ which appear are still non-touching with each other. Hence $u\left(T_{i}\left(v^{\prime}\right)\right)=u^{\prime}\left(v^{\prime}\right)$. We have $v=T_{i}\left(v^{\prime}\right)$ or $v=T_{i}\left(v^{\prime}\right)+e_{i}$. If $v=T_{i}\left(v^{\prime}\right)$, we have $u(v)=u^{\prime}\left(v^{\prime}\right)$, as desired. Assume now that $v=T_{i}\left(v^{\prime}\right)+e_{i}$. From the definition of $\xi_{i}$ we see that either

(i) $[i, i]$ is non-touching with any $\left[a_{s}, b_{s}\right]$, or

(ii) $[i, i]$ is not non-touching with some $[a, b]=\left[a_{s}, b_{s}\right]$ which is uniquely determined and we have $a<i<b$. 
If (i) holds, then $e_{i}$ does not contribute to $u(v)$ and $u(v)=u\left(T_{i}\left(v^{\prime}\right)\right)=u^{\prime}\left(v^{\prime}\right)$. We now assume that (ii) holds. Then $e_{[a, b]}+e_{i}=e_{[a, i-1]}+e_{[i+1, b]}$. We consider six cases.

(1) $a$ is even $b$ is odd, $i$ is even; then $|[i+1, b]|$ is odd so that the contribution of $e_{[a, i-1]}+e_{[i+1, b]}$ to $u(v)$ is $1+0$; this equals the contribution of $e_{[a, b]}$ to $u\left(T_{i}\left(v^{\prime}\right)\right)$ which is 1 .

(2) $a$ is even, $b$ is odd, $i$ is odd; then $|[a, i-1]|$ is odd so that the contribution of $e_{[a, i-1]}+e_{[i+1, b]}$ to $u(v)$ is $0+1$; this equals the contribution of $e_{[a, b]}$ to $u\left(T_{i}\left(v^{\prime}\right)\right)$ which is 1 .

(3) $a$ is odd, $b$ is even, $i$ is even; then $|[i+1, b]|$ is odd so that the contribution of $e_{[a, i-1]}+e_{[i+1, b]}$ to $u(v)$ is $0-1$; this equals the contribution of $e_{[a, b]}$ to $u\left(T_{i}\left(v^{\prime}\right)\right)$ which is -1 .

(4) $a$ is odd, $b$ is even, $i$ is odd; then $|[a, i-1]|$ is odd so that the contribution of $e_{[a, i-1]}+e_{[i+1, b]}$ to $u(v)$ is $-1+0$; this equals the contribution of $e_{[a, b]}$ to $u\left(T_{i}\left(v^{\prime}\right)\right)$ which is -1 .

(5) $a={ }_{2} b={ }_{2} i+1$; then $|[a, i-1]|$ is odd, $|[i+1, b]|$ is odd so that the contribution of $e_{[a, i-1]}+e_{[i+1, b]}$ to $u(v)$ is $0+0$; this equals the contribution of $e_{[a, b]}$ to $u\left(T_{i}\left(v^{\prime}\right)\right)$ which is 0 .

(6) $a={ }_{2} b={ }_{2} i$; then the contribution of $e_{[a, i-1]}+e_{[i+1, b]}$ to $u(v)$ is $1-1$ or $-1+1$; this equals the contribution of $e_{[a, b]}$ to $u\left(T_{i}\left(v^{\prime}\right)\right)$ which is 0 .

This proves (b).

1.12. We view $V$ as the set of vertices of a graph in which $x, x^{\prime}$ in $V$ are joined whenever there exists $i \in[1, D]$ such that $x+x^{\prime}=e_{i},\left(x, e_{i}\right)=\left(x^{\prime}, e_{i}\right)=0$. Similarly if $D \geq 2$, we view $V^{\prime}$ as the set of vertices of a graph in which $y, y^{\prime}$ in $V^{\prime}$ are joined whenever there exists $i \in[1, D-2]$ such that $y+y^{\prime}=e_{i}^{\prime},\left(y, e_{i}^{\prime}\right)^{\prime}=\left(y^{\prime}, e_{i}^{\prime}\right)^{\prime}=0$. We show:

(a) If $y, y^{\prime}$ in $V^{\prime}$ are joined in the graph $V^{\prime}$, then $T_{i}(y), T_{i}\left(y^{\prime}\right)$ are in the same connected component of the graph $V$.

We can find $j \in[1,2 d-2]$ such that $\left(y, e_{j}^{\prime}\right)^{\prime}=\left(y^{\prime}, e_{j}^{\prime}\right)^{\prime}=0, y+y^{\prime}=e_{j}^{\prime}$. Hence $\left(\tilde{y}, T_{i}\left(e_{j}^{\prime}\right)\right)=\left(\tilde{y}^{\prime}, T_{i}\left(e_{j}^{\prime}\right)\right)=0, \tilde{y}+\tilde{y}^{\prime}=T_{i}\left(e_{j}^{\prime}\right)$ where $\tilde{y}=T_{i}(y), \tilde{y}^{\prime}=T_{i}\left(y^{\prime}\right)$. If $T_{i}\left(e_{j}^{\prime}\right)=e_{h}$ for some $h \in[1,2 d]$, then $\tilde{y}, \tilde{y}^{\prime}$ are joined in $V$, as required. If this condition is not satisfied, then $1<i<D, j=i-1$ and $T_{i}\left(e_{j}^{\prime}\right)=e_{j}+e_{j+1}+e_{j+2}$. We have $\left(\tilde{y}, e_{j}+e_{j+1}+e_{j+2}\right)=0, \tilde{y}+\tilde{y}^{\prime}=e_{j}+e_{j+1}+e_{j+2}$. Since $\tilde{y} \in V_{i}$, we have $\left(\tilde{y}, e_{i}\right)=0$ hence $\left(\tilde{y}, e_{j+1}\right)=0$ so that $\left(\tilde{y}, e_{j}\right)=\left(\tilde{y}, e_{j+2}\right)$. We are in one of the two cases below.

(1) We have $\left(\tilde{y}, e_{j}\right)=\left(\tilde{y}, e_{j+2}\right)=0$.

(2) We have $\left(\tilde{y}, e_{j}\right)=\left(\tilde{y}, e_{j+2}\right)=1$.

In case (1) we consider the four term sequence $\tilde{y}, \tilde{y}+e_{j}, \tilde{y}+e_{j}+e_{j+2}, \tilde{y}+e_{j}+$ $e_{j+1}+e_{j+2}=\tilde{y}^{\prime}$; any two consecutive terms of this sequence are joined in the graph $V$. In case (2) we consider the four term sequence $\tilde{y}, \tilde{y}+e_{j+1}, \tilde{y}+e_{j}+e_{j+1}, \tilde{y}+$ $e_{j}+e_{j+1}+e_{j+2}=\tilde{y}^{\prime}$; any two consecutive terms of this sequence are joined in the graph $V$. We see that in both cases $\tilde{y}, \tilde{y}^{\prime}$ are in the same connected component of $V$; (a) is proved.

Let $V_{0}=\{x \in V ; u(x)=0\}$. Note that $0 \in V_{0}$. We show:

(b) If $x \in V_{0}$, then $x, 0$ are in the same component of the graph $V$. 
We argue by induction on $D$. If $D=0$ there is nothing to prove. Assume now that $D \geq 2$. If $\left(x, e_{i}\right)=1$ for all $i \in[1, D]$, then

$$
\begin{gathered}
x=e_{[2,3]}+e_{[6,7]}+e_{[10,11]}+\cdots+e_{[D-2, D-1]} \text { if } D / 2 \text { is even, } \\
x=e_{[1,2]}+e_{[5,6]}+e_{[9,10]}+\cdots+e_{[D-1, D]} \text { if } D / 2 \text { is odd. }
\end{gathered}
$$

In both cases we have $u(x) \neq 0$ contradicting our assumption. Thus we have $\left(x, e_{i}\right)=0$ for some $i \in[1, D]$. By 1.9(a) we have $x=T_{i}\left(x^{\prime}\right)+c e_{i}$ for some $x^{\prime} \in V^{\prime}$ and some $c \in \mathbf{F}_{2}$. By 1.11(b) we have $u^{\prime}\left(x^{\prime}\right)=0$. By the induction hypothesis $x^{\prime}, 0$ are in the same connected component of $V^{\prime}$. By (a), $T_{i}\left(x^{\prime}\right), 0$ are in the same connected component of $V$. Clearly $x, T_{i}\left(x^{\prime}\right)$ are joined in the graph $V$. Hence $x, 0$ are joined in the graph $V$. We see that (b) holds.

We show:

(c) $V_{0}$ is a connected component of the graph $V$.

If $x, x^{\prime}$ in $V$ are in the same connected component of $V$, then $u(x)=u\left(x^{\prime}\right)$. (We can assume that $x, x^{\prime}$ are joined in the graph $V$. Then for some $i \in[1, D]$ we have $x=T_{i}(y)+c e_{i}, x^{\prime}=T_{i}(y)+c^{\prime} e_{i}$ where $y \in V^{\prime}, c \in \mathbf{F}_{2}, c^{\prime} \in \mathbf{F}_{2}$. By 1.11(b) we have $u(x)=u^{\prime}(y), u\left(x^{\prime}\right)=u^{\prime}(y)$, hence $u(x)=u\left(x^{\prime}\right)$, as required.) Thus $V_{0}$ is a union of connected components of $V$. On the other hand, by (b), $V_{0}$ is contained in a connected component of the graph $V$. This proves (c).

\subsection{We show:}

(a) If $B \in S_{D}$, then $\langle B\rangle \subset V_{0}$.

We argue by induction on $D$. If $D=0$ there is nothing to prove. Assume that $D \geq 2$. If $B=\emptyset$ there is nothing to prove. Assume that $B \neq \emptyset$. We can find $i \in[1, D]$ and $B^{\prime} \in S_{D-2}$ such that $B=t_{i}\left(B^{\prime}\right)$. By 1.9(c) we have $\langle B\rangle=T_{i}\left(\left\langle B^{\prime}\right\rangle\right) \oplus \mathbf{F}_{2} e_{i}$. Using 1.11)(b), to prove that $u=0$ on $\langle B\rangle$ it is enough to prove that $u^{\prime}=0$ on $\left\langle B^{\prime}\right\rangle$ and this follows from the induction hypothesis. This proves (a).

We show:

(b) If $x \in V_{0}$, then $x \in\langle B\rangle$ for some $B \in S_{d}$.

We argue by induction on $D$. If $D=0$ there is nothing to prove. Assume that $D \geq 2$. As in the proof of 1.12 (b), from the fact that $u(x)=0$ we can deduce that $\left(x, e_{i}\right)=0$ for some $i \in[1, D]$. By [1.9 (a) we have $x=T_{i}\left(x^{\prime}\right)+c e_{i}$ for some $x^{\prime} \in V^{\prime}$ and some $c \in \mathbf{F}_{2}$. By 1.11(b) we have $u^{\prime}\left(x^{\prime}\right)=0$. By the induction hypothesis we have $x^{\prime} \in\left\langle B^{\prime}\right\rangle$ for some $B^{\prime} \in S_{D-2}$. Then $x \in T_{i}\left(\left\langle B^{\prime}\right\rangle\right) \oplus \mathbf{F}_{2} e_{1}=\langle B\rangle$ (we use 1.9(c)). This proves (b).

From (a),(b) we deduce:

(c) We have $\bigcup_{B \in S_{D}}\langle B\rangle=V_{0}$.

A closely related result is proved in [L3, 3.4].

1.14. The function $\epsilon: S_{D} \rightarrow V$ has values in $\bigcup_{B \in S_{D}}\langle B\rangle$ (see [1.9(c)) hence by 1.13(c) it has values in $V_{0}$. Thus, it can be viewed as a function $\epsilon: S_{D} \rightarrow V_{0}$.

From 1.10(a) we see that:

(a) $\epsilon: S_{D} \rightarrow V_{0}$ is injective.

1.15. Let $F_{0}$ be the $\mathbf{Q}$-vector space consisting of functions $V_{0} \rightarrow \mathbf{Q}$. For $x \in V_{0}$ let $\psi_{x} \in F_{0}$ be the characteristic function of $x$. For $B \in S_{D}$ let $\Psi_{B} \in F_{0}$ be the characteristic function of $\langle B\rangle$. (We use that $\langle B\rangle \subset V_{0}$; see 1.13.) Let $\tilde{F}_{0}$ be the Q-subspace of $F_{0}$ generated by $\left\{\Psi_{B} ; B \in S_{D}\right\}$. When $D \geq 2$ we define $\psi_{x^{\prime}}^{\prime}$ for $x^{\prime} \in V^{\prime}$ and $\Psi_{B^{\prime}}^{\prime}$ for $B^{\prime} \in S_{D-2}, F_{0}^{\prime}, \tilde{F}_{0}^{\prime}$, in terms of $S_{D-2}$ in the same way as 
$\psi_{x}, \Psi_{B}, F_{0}, \tilde{F}_{0}$ were defined in terms of $S_{D}$. For any $i \in[1, D]$ we define a linear map $\theta_{i}: F_{0}^{\prime} \rightarrow F_{0}$ by $f^{\prime} \mapsto f$ where $f\left(T_{i}\left(x^{\prime}\right)+c e_{i}\right)=f^{\prime}\left(x^{\prime}\right)$ for $x^{\prime} \in V^{\prime}, c \in \mathbf{F}_{2}$, $f(x)=0$ for $x \in V-e_{i}^{\perp}$. We have

$\theta_{i}\left(\psi_{x^{\prime}}^{\prime}\right)=\psi_{T_{i}\left(x^{\prime}\right)}+\psi_{T_{i}\left(x^{\prime}\right)+e_{i}}$ for any $x^{\prime} \in V^{\prime}$,

$\theta_{i}\left(\Psi_{B^{\prime}}^{\prime}\right)=\Psi_{t_{i}\left(B^{\prime}\right)}$ for any $B^{\prime} \in S_{D-2}$.

We show:

(a) For any $x \in V_{0}$, we have $\psi_{x} \in \tilde{F}_{0}$.

We argue by induction on $D$. If $D=0$ the result is obvious. We now assume that $D \geq 2$. We first show:

(b) If $x, \tilde{x}$ in $V_{0}$ are joined in the graph $V$ and if (a) holds for $x$, then (a) holds for $\tilde{x}$.

We can find $j \in[1,2 d]$ such that $x+\tilde{x}=e_{j},\left(x, e_{j}\right)=0$. We have $x=T_{j}\left(x^{\prime}\right)+c e_{j}$, $\tilde{x}=T_{j}\left(x^{\prime}\right)+c^{\prime} e_{j}$ where $x^{\prime} \in V^{\prime}$ and $c \in \mathbf{F}_{2}, c^{\prime} \in \mathbf{F}_{2}, c+c^{\prime}=1$. By the induction hypothesis we have $\psi_{x^{\prime}}^{\prime}=\sum_{B^{\prime} \in S_{D-2}} a_{B^{\prime}} \Psi_{B^{\prime}}^{\prime}$ where $a_{B^{\prime}} \in \mathbf{Q}$. Applying $\theta_{j}$ we obtain

$$
\psi_{x}+\psi_{\tilde{x}}=\sum_{B^{\prime} \in S_{D-2}} a_{B^{\prime}} \Psi_{t_{j}\left(B^{\prime}\right)} .
$$

We see that $\psi_{x}+\psi_{\tilde{x}} \in \tilde{F}_{0}$. Since $\psi_{x} \in \tilde{F}$, by assumption, we see that $\psi_{\tilde{x}} \in \tilde{F}$. This proves (b).

We now prove (a). Since $V_{0}$ is the connected component of $V$ containing 0 , to prove (a) it is enough (by (b)) to show that (a) holds when $x=0$. This follows from the fact that $\psi_{0}=\Psi_{B}$ where $B=\emptyset$. This proves (a).

Since $\tilde{F}_{0} \subset F_{0}$, we see that (a) implies:

(c) $F_{0}=\tilde{F}_{0}$.

We have the following result.

Theorem 1.16. (a) $\left\{\Psi_{B} ; B \in S_{D}\right\}$ is a $\mathbf{Q}$-basis of $F_{0}$.

(b) $\epsilon: S_{D} \rightarrow V_{0}$ is a bijection.

Proof. From the definition of $\tilde{F}_{0}$ we have $\operatorname{dim} \tilde{F}_{0} \leq\left|S_{D}\right|$. By 1.14(a) we have $\left|S_{D}\right| \leq\left|V_{0}\right|=\operatorname{dim} F_{0}$. Since $F_{0}=\tilde{F}_{0}$ (see 1.15(c)) it follows that $\operatorname{dim} \tilde{F}_{0}=\left|S_{D}\right|=$ $\left|V_{0}\right|=\operatorname{dim} F_{0}$. Using again the definition of $\tilde{F}_{0}$ and the equality $F_{0}=\tilde{F}_{0}$ we see that (a) holds. Since the map in (b) is injective (see 1.14(a)) and $\left|S_{D}\right|=\left|V_{0}\right|$ we see that it is a bijection so that (b) holds.

1.17. In this subsection we describe the bijection in 1.16 (b) assuming that $D$ is 2 , 4 , or 6 . In each case we give a table in which there is one row for each $B \in S_{D}$; the row corresponding to $B$ is of the form $\langle B\rangle:(\ldots)$ where $B$ is represented by the list of intervals of $B$ (we write an interval such as $[4,6]$ as 456 ) and (...) is a list of the vectors in $\langle B\rangle$ (we write 1235 instead of $e_{1}+e_{2}+e_{3}+e_{5}$, etc.). In each list $(\ldots)$ we single out the vector corresponding $\epsilon(B)$ in 1.16 (b) by putting it in a box. Any non-boxed entry in (...) appears as a boxed entry in some previous row. We see that in these cases, 0.5 (i) holds.

The table for $D=2$.

$\emptyset:(0)$

$\langle 1\rangle:(0,1)$

$\langle 2\rangle:(0,2)$.

The table for $D=4$.

$\emptyset:(0)$ 
$\langle 1\rangle:(0,1)$
$\langle 2\rangle:(0,2)$
$\langle 3\rangle:(0,3)$
$\langle 4\rangle:(0,4)$
$\langle 1,3\rangle:(0,1,3,13)$
$\langle 1,4\rangle:(0,1,4,14)$
$\langle 2,4\rangle:(0,2,4,24)$
$\langle 2,123\rangle:(0,2,13,123)$
$\langle 3,234\rangle:(0,3,24,234)$.

The table for $D=6$.

$\emptyset:(0)$

$\langle 1\rangle:(0,1)$

$\langle 2\rangle:(0,2)$

$\langle 3\rangle:(0,3)$

4\langle\rangle$:(0,4)$

5\langle\rangle$:(0,5)$

$\langle 6\rangle:(0,6)$

$\langle 1,4\rangle:(0,1,4,14)$

$\langle 1,6\rangle:(0,1,6,16)$

$\langle 2,4\rangle:(0,2,4,24)$

$\langle 2,5\rangle:(0,2,5,25)$

$\langle 2,6\rangle:(0,2,6,26)$

$\langle 3,6\rangle:(0,3,6,36)$

$\langle 4,6\rangle:(0,4,6,46)$

$\langle 1,3\rangle:(0,1,3,13)$

$\langle 1,5\rangle:(0,1,5,15)$

$\langle 3,5\rangle:(0,3,5,35)$

$\langle 2,123\rangle:(0,2,13,123)$

$\langle 3,234\rangle:(0,3,24,234)$

$\langle 4,345\rangle:(0,4,35,345)$

$\langle 5,456\rangle:(0,5,46,456)$

$\langle 1,3,5\rangle:(0,1,3,5,13,15,35,135)$

$\langle 1,3,6\rangle:(0,1,3,6,13,16,36,136)$

$\langle 1,4,345\rangle:(0,1,4,345,14,35,135,1345)$

$\langle 1,4,6\rangle:(0,1,4,6,14,16,46,146)$

$\langle 2,4,6\rangle:(0,2,4,6,24,26,46,246)$

$\langle 1,5,456\rangle:(0,1,5,456,15,46,146,1456)$

$\langle 2,5,456\rangle:(0,2,5,456,25,46,246,2456)$

$\langle 2,5,123\rangle:(0,2,5,123,25,13,135,1235)$

$\langle 2,6,123\rangle:(0,2,6,123,26,13,136,1236)$

$\langle 2,4,12345\rangle:(0,2,4,24,1345,1235,135,12345)$ 


$$
\begin{aligned}
& \langle 3,234,12345\rangle:(0,3,234,12345,24,15,135,1245) \\
& \langle 3,6,234\rangle:(0,3,6,234,24,36,246,2346) \\
& \langle 3,5,23456\rangle:(0,3,5,2456,35,2346,246,23456) \\
& \langle 4,345,23456\rangle:(0,4,345,23456,35,26,246,2356) .
\end{aligned}
$$

\section{The $\operatorname{Sets} \mathcal{F}_{*}(V), \mathcal{F}(V)$}

2.1. We no longer assume that $D$ is even. We define a collection $\mathcal{F}_{*}(V)$ and a collection $\mathcal{F}(V)$ of subspaces of $V$ by induction on $D$ as follows. If $D \in\{0,1\}$, $\mathcal{F}_{*}(V)$ and $\mathcal{F}(V)$ consist of $\{0\}$. If $D \geq 2$, a subspace $X$ of $V$ is said to be in $\mathcal{F}_{*}(V)$ if there exists $i \in[1, D]$ (if $D$ is even) or $i \in[1, D-1]$ (if $D$ is odd) and $X^{\prime} \in \mathcal{F}_{*}\left(V^{\prime}\right)$ such that $X=T_{i}\left(X^{\prime}\right) \oplus \mathbf{F}_{2} e_{i}$; a subspace $X$ of $V$ is said to be in $\mathcal{F}(V)$ if either $X=0$ or if there exists $i \in[1, D]$ (if $D$ is even) or $i \in[1, D-1]$ (if $D$ is odd) and $X^{\prime} \in \mathcal{F}\left(V^{\prime}\right)$ such that $X=T_{i}\left(X^{\prime}\right) \oplus \mathbf{F}_{2} e_{i}$. By induction on $D$ we see that for $X \in \mathcal{F}_{*}(V)$ we have $X \in \mathcal{F}(V)$ and $\operatorname{dim}(X)=D / 2$ if $D$ is even, $\operatorname{dim}(X)=(D-1) / 2$ if $D$ is odd. When $D$ is odd, let $\underline{V}$ be the subspace of $V$ with basis $\left\{e_{1}, e_{2}, \ldots, e_{D-1}\right\}$. This vector space with basis is of the same kind as $V$ in 1.9 (but of even dimension) hence $\mathcal{F}(\underline{V}), \mathcal{F}_{*}(\underline{V})$ are defined. Using induction on $D$ we see that for $D$ odd we have $\mathcal{F}(V)=\mathcal{F}(\underline{V}), \mathcal{F}_{*}(V)=\mathcal{F}_{*}(\underline{V})$. Thus, the study of $\mathcal{F}(V), \mathcal{F}_{*}(V)$ when $D$ is odd is reduced to the similar study when $D$ is even.

We now assume that $D$ is even. If $B \in S_{D}$, then $\langle B\rangle \in \mathcal{F}(V)$ (this follows from 1.9( (c) by induction on $D)$. Conversely, if $X \in \mathcal{F}(V)$, then there exists $B \in S_{D}$ such that $X=\langle B\rangle$ (this again follows from 1.9(c) by induction on $D$ ). Thus we have a surjective map $S_{D} \rightarrow \mathcal{F}(V), B \mapsto\langle B\rangle$. We show:

(a) This map is a bijection.

Indeed, if $B, \tilde{B}$ in $S_{D}$ satisfy $\langle B\rangle=\langle\tilde{B}\rangle$, then the functions $\Psi_{B}, \Psi_{\tilde{B}}$ in $F_{0}$ coincide hence $B=\tilde{B}$ by 1.16(a). This proves (a).

For $B \in S_{D}$ we show:

(b) $\left\{e_{I} ; I \in B\right\}$ is an $\mathbf{F}_{2}$-basis of $\langle B\rangle$.

We argue by induction on $D$. If $D=0$ there is nothing to prove. Assume that $D \geq 2$. If $B=\emptyset$, then (b) is obvious. We now assume that $B \neq \emptyset$. Assume that $\sum_{I \in B} c_{I} e_{I}=0$ with $c_{I} \in \mathbf{F}_{2}$ not all zero. We can find $I=[a, b] \in B$ with $c_{I} \neq 0$ and $|I|$ maximal. If $I^{\prime} \in B$ is such that $a \in I^{\prime}, I^{\prime} \neq I, c_{I^{\prime}} \neq 0$, then by $\left(P_{0}\right)$ we have $I \prec I^{\prime}$ (contradicting the maximality of $|I|$ ) or $I^{\prime} \prec I$ (contradicting $\left.a \in I^{\prime}\right)$. Thus no $I^{\prime}$ as above exists. Thus when $\sum_{I_{1} \in B} c_{I_{1}} e_{I_{1}}$ is written in the basis $\left\{e_{j} ; j \in[1, D]\right\}$, the coefficient of $e_{a}$ is $c_{I_{1}}$ hence $c_{I_{1}}=0$, contradicting $c_{I_{1}} \neq 0$. This proves (b).

We show:

(c) If $X \in \mathcal{F}(V)$, then $X$ is an isotropic subspace of $V$.

We argue by induction on $D$. If $D=0$ there is nothing to prove. Assume that $D \geq 2$. If $X=0$, then (c) is obvious. We now assume that $X \neq 0$. Then there exists $i \in[1, D]$ and $X^{\prime} \in \mathcal{F}\left(V^{\prime}\right)$ such that $X=T_{i}\left(X^{\prime}\right) \oplus \mathbf{F}_{2} e_{i}$. By the induction hypothesis, $X^{\prime}$ is isotropic in $V^{\prime}$. Since $T_{i}$ is compatible with the symplectic forms it follows that $T_{i}\left(X^{\prime}\right)$ is an isotropic subspace of $V$. Since $T_{i}\left(X^{\prime}\right)$ is contained in $e_{i}^{\perp}$, $T_{i}\left(X^{\prime}\right) \oplus \mathbf{F}_{2} e_{i}$ is also isotropic. This proves (c). Alternatively, (c) can be deduced from property $\left(P_{0}\right)$. 
2.2. For $\delta \in\{0,1\}$ let $[1, D]^{\delta}=\left\{i \in[1, D] ; i={ }_{2} \delta\right\}$. Let $V^{\delta}$ be the subspace of $V$ with basis $\left\{e_{i} ; i \in[1, D]^{\delta}\right\}$. We have $V=V^{0} \oplus V^{1}$. Similarly, if $D \geq 2$, we have $V^{\prime}=V^{\prime 0} \oplus V^{\prime 1}$ where $V^{\prime \delta}$ has basis $\left\{e_{i}^{\prime} ; i \in[1, D-2]^{\delta}\right\}$.

For any $I \in \mathcal{I}_{D}^{1}$ and $\delta \in\{0,1\}$ we set $I^{\delta}=I \cap[1, D]^{\delta}$, so that $I=I^{0} \sqcup I^{1}$; we define $\kappa(I) \in\{0,1\}$ by $a={ }_{2} \kappa(I)$ or equivalently $b={ }_{2} \kappa(I)$ where $I=[a, b]$. We show:

(a) Let $B \in S_{D}$ and let $I \in B$. Let $\delta=\kappa(I)$. We have $e_{I^{\delta}}=\sum_{I^{\prime} \in B ; I^{\prime} \subset I} e_{I^{\prime}}$.

We argue by induction on $|I|$. If $|I|=1$ the result is obvious. Assume now that $|I|>1$. By $\left(P_{0}\right),\left(P_{1}\right)$, we can find $\left[a_{1}, b_{1}\right],\left[a_{2}, b_{2}\right], \ldots,\left[a_{k}, b_{k}\right]$ in $B$ such that $a_{1} \leq b_{1}<a_{2} \leq b_{2}<a_{3} \leq b_{3}<, \ldots, a_{1}, b_{1}, a_{2}, b_{2}, \ldots$, are all in $1-\delta+2 \mathbf{Z}$ and $[a, b] \cap(1-\delta+2 \mathbf{Z}) \subset\left[a_{1}, b_{1}\right] \cup\left[a_{2}, b_{2}\right] \cup \cdots \cup\left[a_{k}, b_{k}\right]$. From the definition we have $e_{I^{\delta}}=e_{I}+\sum_{j=1}^{k} e_{\left[a_{j}, b_{j}\right]^{1-\delta}}$. By the induction hypothesis, for $j \in[1, k]$ we have $e_{\left[a_{j}, b_{j}\right]^{1-\delta}}=\sum_{I^{\prime} \in B ; I^{\prime} \subset\left[a_{j}, b_{j}\right]} e_{I^{\prime}}$. Thus we have

$$
e_{I^{\delta}}=e_{I}+\sum_{I^{\prime} \in B ; I^{\prime} \subset \cup_{j}\left[a_{j}, b_{j}\right]} e_{I^{\prime}}=\sum_{I^{\prime} \in B ; I^{\prime} \subset I} e_{I^{\prime}} .
$$

This proves (a).

We show:

(b) Let $B \in S_{D}$. Then $\left\{e_{I^{\kappa(I)}} ; I \in B\right\}$ is a basis of the vector space $\langle B\rangle$.

From (a) we see that the collection of vectors $\left\{e_{I^{\kappa(I)}} ; I \in B\right\}$ is related to the collection of vectors $\left\{e_{I} ; I \in B\right\}$ by an upper triangular matrix with 1 on the diagonal. Hence the result follows from 2.1(b).

We deduce that if $B \in S_{D}$ and $X=\langle B\rangle \in \mathcal{F}(V)$, then for $\delta \in\{0,1\}$,

(c) $X^{\delta}=X \cap V^{\delta}$ has basis $\left\{e_{I^{\kappa(I)}} ; I \in B, \kappa(I)=\delta\right\}$; in particular, $X=X^{0} \oplus X^{1}$.

2.3. Assume that $D \geq 2$. Let $i \in[1, D]$ and let $\delta \in\{0,1\}$. There is a unique linear map $T_{i}^{\delta}: V^{\prime \delta} \rightarrow V^{\delta}$ such that

$T_{i}^{\delta}\left(e_{k}^{\prime}\right)=e_{k}$ if $k \leq i-2, k={ }_{2} \delta$;

$T_{i}^{\delta}\left(e_{k}^{\prime}\right)=e_{k+2}$ if $k \geq i, k={ }_{2} \delta$;

$T_{i}^{\delta}\left(e_{i-1}^{\prime}\right)=e_{i-1}+e_{i+1}$ if $i={ }_{2} \delta+1,1<i<D$.

Note that $T_{i}^{\delta}$ is injective and $(x, y)^{\prime}=\left(T_{i}^{0}(x), T_{i}^{1}(y)\right)$ for any $x \in V^{\prime 0}, y \in V^{\prime 1}$. For any $I^{\prime} \in \mathcal{I}_{D-2}^{1}$ such that $\kappa\left(I^{\prime}\right)=\delta$ we have $T_{i}^{\delta}\left(e_{I^{\prime} \delta}^{\prime}\right)=e_{\xi_{i}\left(I^{\prime}\right)^{\delta}}$. (Here $\kappa\left(I^{\prime}\right), I^{\prime \delta}$ are defined in terms of $I^{\prime}$ in the same way as $\kappa(I), I^{\delta}$ are defined in 2.2.) Let $V_{i}^{\delta}$ be the image of $T_{i}^{\delta}: V^{\prime \delta} \rightarrow V^{\delta}$. From the definitions we deduce:

(a) We have $V_{i} \oplus \mathbf{F}_{2} e_{i}=V_{i}^{0} \oplus V_{i}^{1} \oplus \mathbf{F}_{2} e_{i}$.

We define a collection $\mathcal{C}\left(V^{\delta}\right)$ of subspaces of $V^{\delta}$ by induction on $D$ as follows. If $D=0, \mathcal{C}\left(V^{\delta}\right)$ consists of $\{0\}$. If $D \geq 2$, a subspace $\mathcal{L}$ of $V^{\delta}$ is said to be in $\mathcal{C}\left(V^{\delta}\right)$ if either $\mathcal{L}=0$ or if there exists $i \in[1, D]$ and $\mathcal{L}^{\prime} \in \mathcal{C}\left(V^{\prime \delta}\right)$ such that $\mathcal{L}=T_{i}^{\delta}\left(\mathcal{L}^{\prime}\right) \oplus \mathbf{F}_{2} e_{i}$ (if $i={ }_{2} \delta$ ) or $\mathcal{L}=T_{i}^{\delta}\left(\mathcal{L}^{\prime}\right)$ (if $i={ }_{2} \delta+1$ ).

We show:

(b) If $X \in \mathcal{F}(V)$, then $X^{\delta} \in \mathcal{C}\left(V^{\delta}\right)$.

We argue by induction on $D$. If $D=0$ the result is obvious. Assume now that $D \geq 2$. If $X=0$ there is nothing to prove. Assume that $X \neq 0$. We can find $i \in[1, D]$ and $X^{\prime} \in \mathcal{F}\left(V^{\prime}\right)$ such that $X=T_{i}\left(X^{\prime}\right) \oplus \mathbf{F}_{2} e_{i}$. By the induction hypothesis we have $X^{\prime \delta} \in \mathcal{C}\left(V^{\prime \delta}\right)$. Hence $T_{i}^{\delta}\left(X^{\prime \delta}\right) \oplus \mathbf{F}_{2} e_{i} \in \mathcal{C}\left(V^{\delta}\right)$ if $i={ }_{2} \delta$, $T_{i}^{\delta}\left(X^{\prime \delta}\right) \in \mathcal{C}\left(V^{\delta}\right)$ if $i={ }_{2} \delta+1$. It is enough to prove that $T_{i}^{\delta}\left(X^{\prime \delta}\right) \oplus \mathbf{F}_{2} e_{i}=X^{\delta}$ if $i={ }_{2} \delta, T_{i}^{\delta}\left(X^{\prime \delta}\right)=X^{\delta}$ if $i={ }_{2} \delta+1$, or that $T_{i}^{\delta}\left(X^{\prime \delta}\right) \oplus \mathbf{F}_{2} e_{i}=\left(T_{i}\left(X^{\prime}\right) \oplus \mathbf{F}_{2} e_{i}\right) \cap V^{\delta}$ 
if $i={ }_{2} \delta, T_{i}^{\delta}\left(X^{\prime \delta}\right)=\left(T_{i}\left(X^{\prime}\right) \oplus \mathbf{F}_{2} e_{i}\right) \cap V^{\delta}$ if $i={ }_{2} \delta+1$. This follows by comparing the definition of $T_{i}^{\delta}$ with that of $T_{i}$.

2.4. Let $\delta \in\{0,1\}$. If $Z$ is a subspace of $V^{\delta}$ we set $Z^{!}=\left\{x \in V^{1-\delta} ;(x, z)=\right.$ $0 \forall z \in Z\}$. Similarly, if $Z^{\prime}$ is a subspace of $V^{\prime \delta}$ we set $Z^{\prime !}=\left\{x \in V^{\prime 1-\delta} ;(x, z)^{\prime}=\right.$ $\left.0 \forall z \in Z^{\prime}\right\}$. Let $\mathcal{L} \in \mathcal{C}\left(V^{\delta}\right)$. We show:

(a) We have $\mathcal{L}^{!} \in \mathcal{C}\left(V^{1-\delta}\right)$ and $\mathcal{L} \oplus \mathcal{L}^{!} \subset V$ is in $\mathcal{F}(V)$.

The first statement of (a) follows from the second statement, using 2.3(b). We prove the second statement of (a) by induction on $D$. If $D=0$ the result is immediate. Assume now that $D \geq 2$. If $\mathcal{L}=0$, then $\mathcal{L}^{!}=V^{1-\delta}=\langle B\rangle$ where $B=$ $\left\{\{j\} ; j \in[1, D]^{1-\delta}\right\} \in S_{D}$; thus we have $\mathcal{L}^{!} \in \mathcal{F}(V)$. Next we assume that $\mathcal{L} \neq 0$. We can find $i \in[1, D]$ and $\mathcal{L}^{\prime} \in \mathcal{C}\left(V^{\prime \delta}\right)$ such that $\mathcal{L}=T_{i}^{\delta}\left(\mathcal{L}^{\prime}\right) \oplus \mathbf{F}_{2} e_{i}$ (if $i={ }_{2} \delta$ ) or $\mathcal{L}=T_{i}^{\delta}\left(\mathcal{L}^{\prime}\right)\left(\right.$ if $\left.i={ }_{2} \delta+1\right)$. By the induction hypothesis we have $\mathcal{L}^{\prime} \oplus \mathcal{L}^{\prime !} \in \mathcal{F}\left(V^{\prime}\right)$. Hence $T_{i}\left(\mathcal{L}^{\prime} \oplus \mathcal{L}^{\prime !}\right) \oplus \mathbf{F}_{2} e_{i} \in \mathcal{F}(V)$. From the definition we have $T_{i}\left(\mathcal{L}^{\prime} \oplus \mathcal{L}^{\prime !}\right) \oplus \mathbf{F}_{2} e_{i}=$ $T_{i}^{\delta}\left(\mathcal{L}^{\prime}\right) \oplus T_{i}^{1-\delta}\left(\mathcal{L}^{\prime !}\right) \oplus \mathbf{F}_{2} e_{i}$. Thus we have $T_{i}^{\delta}\left(\mathcal{L}^{\prime}\right) \oplus T_{i}^{1-\delta}\left(\mathcal{L}^{\prime !}\right) \oplus \mathbf{F}_{2} e_{i} \in \mathcal{F}(V)$ or equivalently $\mathcal{L} \oplus T_{i}^{1-\delta}\left(\mathcal{L}^{\prime !}\right) \in \mathcal{F}(V)$ (if $i={ }_{2} \delta$ ) and $\mathcal{L} \oplus T_{i}^{1-\delta}\left(\mathcal{L}^{\prime !}\right) \oplus \mathbf{F}_{2} e_{i} \in \mathcal{F}(V)$ (if $\left.i={ }_{2} \delta+1\right)$. It is enough to show: $\mathcal{L}^{!}=T_{i}^{1-\delta}\left(\mathcal{L}^{\prime !}\right)$ if $i={ }_{2} \delta$ and $\mathcal{L}^{!}=T_{i}^{1-\delta}\left(\mathcal{L}^{\prime !}\right) \oplus \mathbf{F}_{2} e_{i}$ if $i={ }_{2} \delta+1$. If $y \in \mathcal{L}^{\prime !}, x \in \mathcal{L}^{\prime}$, we have $\left(T_{i}^{1-\delta}(y), T_{i}^{\delta}(x)\right)=(y, x)^{\prime}=0$; if $i={ }_{2} \delta$ we have $\left(T_{i}^{1-\delta}(y), e_{i}\right)=0$. If $i={ }_{2} \delta+1$ we have $\left(e_{i}, T_{i}^{\delta}(x)\right)=0$. We see that $T_{i}^{1-\delta}\left(\mathcal{L}^{\prime !}\right) \subset \mathcal{L}^{!}$if $i={ }_{2} \delta$ and $T_{i}^{1-\delta}\left(\mathcal{L}^{\prime !}\right) \oplus \mathbf{F}_{2} e_{i} \subset \mathcal{L}^{!}$if $i={ }_{2} \delta+1$. The last two inclusions are between vector spaces of the same dimension; hence they must be equalities. This completes the proof of (a).

Let $S_{D, *}=\left\{B \in S_{D} ;|B|=D / 2\right\}$. From 2.1(b) we see that the bijection $S_{D} \stackrel{\sim}{\longrightarrow} \mathcal{F}(V), B \mapsto\langle B\rangle$ (see 2.1 (a)) restricts to a bijection

(b) $S_{D, *} \stackrel{\sim}{\longrightarrow} \mathcal{F}_{*}(V)$.

We show:

(c) We have a bijection $\iota: \mathcal{C}\left(V^{\delta}\right) \stackrel{\sim}{\rightarrow} \mathcal{F}_{*}(V)$ given by $\iota(\mathcal{L})=\mathcal{L} \oplus \mathcal{L}^{\text {! }}$.

The fact that $\iota$ is well defined follows from (a). (For $\mathcal{L} \in \mathcal{C}\left(V^{\delta}\right)$ we have $\operatorname{dim}\left(\mathcal{L} \oplus \mathcal{L}^{!}\right)=D / 2$.) We define $\iota^{\prime}: \mathcal{F}_{*}(V) \rightarrow \mathcal{C}\left(V^{\delta}\right)$ by $X \mapsto X^{\delta}$. This is well defined by 2.3 (b). Clearly, $\iota^{\prime} \iota=1$. Let $X \in \mathcal{F}_{*}(V)$. Then $X^{1-\delta} \subset\left(X^{\delta}\right)^{!}$since $X$ is isotropic so that $X^{\delta} \oplus\left(X^{\delta}\right)^{!} \subset X$; this is an inclusion of vector spaces of the same dimension, hence is an equality. Thus $\iota \iota^{\prime}=1$. This proves that $\iota$ is a bijection.

2.5. Let $\delta \in\{0,1\}$. We define a subset $S_{D}^{\delta}$ of $R_{D}^{1}$ by induction on $D$ as follows. When $D=0, S_{D}^{\delta}$ consists of $\emptyset \in R_{D}^{1}$. When $D \geq 2$ we say that $\beta \in R_{D}^{1}$ is in $S_{D}^{\delta}$ if either $\beta=\emptyset$ or if

(i) there exists $i \in[1, D]$ and $\beta^{\prime} \in S_{D-2}^{\delta}$ such that $\beta=\left\{\xi_{i}\left(I^{\prime}\right) ; I^{\prime} \in \beta^{\prime}\right\} \sqcup\{i\}$ if $i={ }_{2} \delta$ and $\beta=\left\{\xi_{i}\left(I^{\prime}\right) ; I^{\prime} \in \beta^{\prime}\right\}$ if $i={ }_{2} \delta+1$.

From the definition we see by induction on $D$ that if $\beta \in S_{D}^{\delta}$ and $I \in \beta$, then $\kappa(I)=\delta$.

Let $S_{D}^{\prime} \delta$ be the set of all $\beta \in R_{D}^{1}$ such that $\kappa(I)=\delta$ for any $I \in \beta$ and such that the following holds:

$\left(P_{0}^{\delta}\right)$ If $I \in \beta, \tilde{I} \in \beta$, then either $I=\tilde{I}$, or $I \boldsymbol{\leftrightarrow} \tilde{I}$, or $I \prec \tilde{I}$, or $\tilde{I} \prec I$.

By arguments similar to those in 1.3 we see that

(a) We have $S_{D}^{\delta}=S_{D}^{\prime}{ }^{\delta}$.

We show:

(b) If $B \in S_{D}$, then ${ }^{\delta} B:=\{I \in B ; \kappa(I)=\delta\}$ is in $S_{D}^{\delta}$.

From 2.5 (c) we see that ${ }^{\delta} B \in S_{D}^{\prime} \delta$ hence (using (a)) ${ }^{\delta} B \in S_{D}^{\delta}$.

Using the definitions we can verify: 
(c) Assume that $D \geq 2$, that $B^{\prime} \in S_{D-2}$, and that $B=t_{i}\left(B^{\prime}\right) \in S_{D}$. Let $\beta^{\prime}={ }^{\delta} B^{\prime} \in S_{D-2}^{\delta}, \beta={ }^{\delta} B \in S_{D}^{d}$. Then $\beta$ is obtained from $\beta^{\prime}$ as in (i) above.

Let ${ }^{\prime} S_{D}^{\delta}$ be the set of all subsets of $R_{D}^{1}$ of the form ${ }^{\delta} B$ for some $B \in S_{D, *}$. We show:

(d) ${ }^{\prime} S_{D}^{\delta}=S_{D}^{\delta}$.

The inclusion ' $S_{D}^{\delta} \subset S_{D}^{\delta}$ follows from (b). Conversely we show that if $\beta \in S_{D}^{\delta}$, then $\beta \in{ }^{\prime} S_{D}^{\delta}$. We argue by induction on $D$. When $D=0$ there is nothing to prove. Assume that $D \geq 2$. If $\beta=\emptyset$ there is nothing to prove. Assume that $\beta \neq \emptyset$. We can find $i \in[1, D]$ and $\beta^{\prime} \in S_{D-2}^{\delta}$ such that $\beta$ is obtained from $\beta^{\prime}$ as in (i) above. By the induction hypothesis we have $\beta^{\prime}={ }^{\delta} B^{\prime}$ where $B^{\prime} \in S_{D-2, *}$. Let $B=t_{i}\left(B^{\prime}\right)$. We have $B \in S_{D, *}$. Let $\tilde{\beta}={ }^{\delta} B \in{ }^{\prime} S_{D}^{\delta}$. By (c), $\tilde{\beta}$ is obtained from $\beta^{\prime}$ as in (i) above. Since $\beta$ has the same property, we have $\tilde{\beta}=\beta$. Thus $\beta \in{ }^{\prime} S_{D}^{d}$, as required. This proves $(d)$.

We show:

(e) The map $S_{D, *} \rightarrow{ }^{\prime} S_{D}^{\delta}, B \mapsto{ }^{\delta} B$ is a bijection.

It is enough to show that this map is injective. Assume that $B \in S_{D, *}, \tilde{B} \in S_{D, *}$ satisfy ${ }^{\delta} B={ }^{\delta} \tilde{B}$. We must show that $B=\tilde{B}$. By the proof of 2.4 (c) we have a bijection $\iota^{\prime}: \mathcal{F}_{*}(V) \rightarrow \mathcal{C}\left(V^{\delta}\right)$ given by $X \mapsto X^{\delta}$. Now $\iota^{\prime}(\langle B\rangle)$ has basis $\left\{e_{I^{\kappa(I)}} ; I \in\right.$ $B, \kappa(I)=\delta\}$ and $\iota^{\prime}(\langle\tilde{B}\rangle)$ has basis $\left\{e_{I^{\kappa(I)}} ; I \in \tilde{B}, \kappa(I)=\delta\right\}$. Since ${ }^{\delta} B={ }^{\delta} \tilde{B}$, these two bases coincide hence $\iota^{\prime}(\langle B\rangle)=\iota^{\prime}(\langle\tilde{B}\rangle)$. Since $\iota^{\prime}$ is a bijection we deduce that $\langle B\rangle=\langle\tilde{B}\rangle$. Using 2.1 (a) we see that $B=\tilde{B}$. This proves (e).

Combining (d),(e) we obtain:

(f) The map $S_{D *} \rightarrow S_{D}^{\delta}, B \mapsto{ }^{\delta} B$ is a bijection.

For any $\beta \in S_{D}^{\delta}$ let $\langle\beta\rangle$ be the $\mathbf{F}_{2^{-}}$-subspace of $V^{\delta}$ spanned by $\left\{e_{I^{\kappa(i)}} ; I \in \beta\right\}$. By the proof of $(\mathrm{e})$, we have $\langle\beta\rangle \in \mathcal{C}\left(V^{\delta}\right)$ and $\operatorname{dim}\langle\beta\rangle=|\beta|$. We show:

(g) The map $\beta \mapsto\langle\beta\rangle$ is a bijection $\iota^{\prime \prime}: S_{D}^{\delta} \stackrel{\sim}{\longrightarrow} \mathcal{C}\left(V^{\delta}\right)$.

We have a commutative diagram

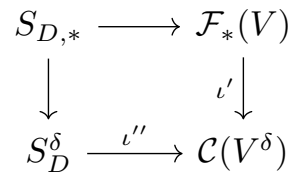

where the top horizontal map is a bijection as in 2.4(b), the left vertical map is a bijection as in (e) (see also (d)), and $\iota^{\prime}$ is a bijection as in the proof of (e). It follows that $\iota^{\prime \prime}$ is a bijection. This proves $(\mathrm{g})$.

2.6. Let $\delta \in\{0,1\}$. We define a bijection $S_{D}^{\delta} \stackrel{\sim}{\rightarrow} S_{D}^{1-\delta}, \beta \rightarrow \beta^{!}$as follows. Let $\beta \in S_{D}^{\delta}$. By 2.5(g), we have $\langle\beta\rangle \in \mathcal{C}\left(V^{\delta}\right)$ and by 2.4(a) we have $\langle\beta\rangle^{!} \in \mathcal{C}\left(V^{1-\delta}\right)$. Then $\beta^{!}$is the unique element of $S_{D}^{1-\delta}$ such that $\langle\beta\rangle^{!}=\left\langle\beta^{!}\right\rangle$; see 2.5)(g). From the definition we have $\left(\beta^{!}\right)^{!}=\beta$ and $\left|\beta^{!}\right|=(D / 2)-|\beta|$. Recall that $\langle\beta\rangle \oplus\left\langle\beta^{!}\right\rangle=\langle B\rangle$ where $B \in S_{D, *}$ satisfies ${ }^{\delta} B=\beta,{ }^{1-\delta} B=\beta$ ! .

The order reversing involution $i \mapsto i^{*}=D+1-i$ of $[1, D]$ induces an involution $R_{D}^{1} \rightarrow R_{D}^{1}, I \mapsto I^{*}=\left\{i^{*} ; i \in I\right\}$ and an involution $S_{D} \rightarrow S_{D}, B \mapsto B^{*}:=\left\{I^{*} ; I \in\right.$ $B\}$. It also induces a bijection $\gamma_{\delta}: S_{D}^{1-\delta} \stackrel{\sim}{\rightarrow} S_{D}^{\delta}$. Then $\beta \mapsto \gamma_{\delta}\left(\beta^{!}\right)$is a bijection $S_{D}^{\delta} \rightarrow S_{D}^{\delta}$ which carries any subset with $m$ elements $(m \in[0, D / 2])$ to a subset with $(D / 2)-m$ elements. 
2.7. Let $\delta \in\{0,1\}$. Let $U^{\delta}=\left\{\left(\mathcal{L}, \mathcal{L}^{\prime}\right) \in \mathcal{C}\left(V^{\delta}\right) \times \mathcal{C}\left(V^{\delta}\right) ; \mathcal{L} \subset \mathcal{L}^{\prime}\right\}$. We define a map (a) $\mathcal{F}(V) \rightarrow U^{\delta}$ by $X \mapsto\left(X^{\delta},\left(X^{1-\delta}\right)^{!}\right)$.

(We have $X^{\delta} \subset\left(X^{1-\delta}\right)$ ! since $X$ is isotropic.) This map is injective since $X$ can be reconstructed from $X^{\delta}, X^{1-\delta}$ : we have $X=X^{\delta} \oplus X^{1-\delta}$.

We note that the map (a) is not surjective. For example, if $D=2, \delta=0$ and $\mathcal{L}=0, \mathcal{L}^{\prime}=\mathbf{F}_{2} e_{2}$, then $\left(\mathcal{L}, \mathcal{L}^{\prime}\right) \in U^{0}$ is not in the image of the map (a). The following result is a reformulation of 2.4 (c).

(b) The map (a) restricts to a bijection $\mathcal{F}_{*}(V) \stackrel{\sim}{\rightarrow}\left\{\left(\mathcal{L}, \mathcal{L}^{\prime}\right) \in U^{\delta} ; \mathcal{L}=\mathcal{L}^{\prime}\right\}$.

2.8. In the remainder of this section we prove Theorem 0.4 assuming that $W$ is a Weyl group of type $B_{n}, C_{n}$, or $D_{n}$. If $|c|=1$ the theorem is trivial; we have $\mathcal{G}_{c}=\{1\}$ and $\mathbf{B}_{c}$ consists of the unique representation in $c$. Assume now that $|c| \geq 2$. As in [L5, 4.5,4.6], [L4], [L6], we can find $D \in\{2,4,6, \ldots\}$ and $\delta \in\{0,1\}$ such that if $V$ is the $\mathbf{F}_{2}$-vector space with basis $\left\{e_{i} ; i \in[1, D]\right\}$ as in 1.9 , then (i)-(iii) below hold.

(i) The group $\mathcal{G}_{c}$ in 0.3 is $V^{\delta}$; hence $M\left(\mathcal{G}_{c}\right)=V^{\delta} \oplus \operatorname{Hom}\left(V^{\delta}, \mathbf{C}^{*}\right)$ can be identified with $V=V^{\delta} \oplus V^{1-\delta}$ (an element $y \in V^{1-\delta}$ can be identified with the homomorphism $V^{\delta} \rightarrow \mathbf{C}^{*}$ given by $\left.x \mapsto(-1)^{(x, y)}\right)$.

(ii) $c$ is naturally in bijection with $V_{0}$ (see 1.12); hence any object $\mathcal{E} \in \mathcal{R}_{c}$ can be viewed as the function $f_{\mathcal{E}}: V_{0} \rightarrow \mathbf{N}$ such that for $E \in c$ the multiplicity of $E$ in $\mathcal{E}$ is equal to the value of $f_{\mathcal{E}}$ at the point of $V_{0}$ corresponding to $E$.

(iii) The constructible representations in $\mathcal{R}_{c}$ viewed as functions $V_{0} \rightarrow \mathbf{N}$ are exactly the characteristic functions of the subsets $X \subset V$ with $X \in \mathcal{F}_{*}(V)$.

(More accurately, the results in [L4] [L6] for $W$ of type $D_{n}$ are formulated in terms of a $V$ as in 1.9 with odd $D$, but they can be restated in terms of a $V$ as in 1.9 with $D$ even, by the argument in the first part of 2.1.)

If $\mathcal{L}$ is a subspace of $V^{\delta}$, then $S_{\mathcal{L}, \mathcal{L}} \in \mathbf{C}\left[M\left(\mathcal{G}_{c}\right)\right]=\mathbf{C}[V]$ (see (i) and 0.2 ) can be identified with the function $V \rightarrow \mathbf{C}$ whose value is 1 at any element of $\mathcal{L} \oplus \mathcal{L}^{!}$and is 0 at any element of $V-\left(\mathcal{L} \oplus \mathcal{L}^{!}\right)$. If $\mathcal{L} \in \mathcal{C}\left(V^{\delta}\right)$ this is the characteristic function of some $X \in \mathcal{F}_{*}(V)$ namely, $X=\mathcal{L} \oplus \mathcal{L}^{!}$; the converse also holds. We see that $\mathfrak{F}_{c}$ (see $0.3)$ consists of the subspaces $\mathcal{L} \in \mathcal{C}\left(V^{\delta}\right)$. We have $0 \in \mathcal{C}\left(V^{\delta}\right)$ hence $\hat{\mathfrak{F}}_{c}=\mathfrak{F}_{c}$. Now $\tilde{\Theta}_{c}$ becomes the set of pairs $\left(\mathcal{L}, \mathcal{L}^{\prime}\right) \in \mathcal{C}\left(V^{\delta}\right) \times \mathcal{C}\left(V^{\delta}\right)$ such that $\mathcal{L} \subset \mathcal{L}^{\prime}$. We define $\Theta_{c}$ to be the set of pairs $\left(\mathcal{L}, \mathcal{L}^{\prime}\right) \in \mathcal{C}\left(V^{\delta}\right) \times \mathcal{C}\left(V^{\delta}\right)$ such that $\mathcal{L} \oplus \mathcal{L}^{\prime !} \in \mathcal{F}(V)$. (We then automatically have $\mathcal{L} \subset \mathcal{L}^{\prime}$ since the subspaces in $\mathcal{F}(V)$ are isotropic. Thus $\Theta_{c} \subset \tilde{\Theta}_{c}$.) If $\left(\mathcal{L}, \mathcal{L}^{\prime}\right) \in \tilde{\Theta}_{c}$, then $S_{\mathcal{L}, \mathcal{L}^{\prime}} \in \mathbf{C}\left[M\left(\mathcal{G}_{c}\right)\right]=\mathbf{C}[V]$ (see (i) and 0.2) can be identified with the function $V \rightarrow \mathbf{C}$ whose value is 1 at any element of $\mathcal{L} \oplus \mathcal{L}^{\prime}$ ! and is 0 at any element of $V-\left(\mathcal{L} \oplus \mathcal{L}^{\prime !}\right)$. If $\left(\mathcal{L}, \mathcal{L}^{\prime}\right) \in \Theta_{c}$, this is the characteristic function of some $X \in \mathcal{F}(V)$, namely $X=\mathcal{L} \oplus \mathcal{L}^{\prime !}$; the converse also holds. We see that $\Theta_{c}$ can be identified with $\mathcal{F}(V)$. With these identifications Theorem 0.4 follows from the results in $₫ 1$ and $₫ 2$. The representations in $\mathbf{B}_{c}$ corespond as in (ii) to the functions $f^{X}: V_{0} \rightarrow \mathbf{N}$ which equal 1 on $X$ and equal 0 on $V_{0}-X$ (where $X \in \mathcal{F}(V))$. The bijection $c \rightarrow \mathbf{B}_{c}$ mentioned in 0.5 is $x \mapsto\left\langle\epsilon^{-1}(x)\right\rangle$ where $\epsilon$ is as in 1.16 (b).

\section{Exceptional Weyl Groups}

3.1. In this section we will prove Theorem 0.4 assuming that $W$ is of exceptionaltype. In 3.23 .8 we will give a table of new representations in $\mathcal{R}_{c}$ in the form of a matrix $M_{c}$ indexed by $c \times c$. (The table will be justified in 3.10) The columns of 
$M_{c}$ are indexed by the representations in $c$. The rows of $M_{c}$ are also indexed by the representations in $c$ (for any $k \in[1,|c|]$, the $k$ th row from up to down is indexed by the same representation in $c$ as the $k$ th column from left to right). Each row of $M_{c}$ corresponds to a new representation; the entries of that row give the multiplicities of the various representations in $c$ in the new representation. The first row in $M_{c}$ stands for the special representation in $c$.

3.2. If $|c|=1, M_{c}$ is the $1 \times 1$ matrix with entry 1 .

3.3. If $|c|=2$ (so that $W$ is of type $E_{7}$ or $E_{8}$ ) we order $c$ using its bijection with $\{(1,1),(1, \epsilon)\}$ in $[\mathrm{L} 5,4.12,4.13]$ (ordered from left to right); then $M_{c}$ is

$$
\left(\begin{array}{ll}
1 & 0 \\
1 & 1
\end{array}\right)
$$

The second row stands for a constructible representation.

3.4. If $|c|=3$ we order $c$ using its bijection with $\left\{(1,1),\left(g_{2}, 1\right),(1, \epsilon)\right\}$ in [L5, 4.10, $4.11,4.12,4.13]$ (ordered from left to right); then $M_{c}$ is

$$
\left(\begin{array}{lll}
1 & 0 & 0 \\
1 & 1 & 0 \\
1 & 0 & 1
\end{array}\right) .
$$

The last two rows stand for constructible representations.

3.5. If $|c|=4$ (so that $W$ is of type $G_{2}$ ) we order $c$ using its bijection with $\left\{(1,1),(1, r),\left(g_{2}, 1\right),\left(g_{3}, 1\right)\right\}$ in [L5, 4.8] (ordered from left to right); then $M_{c}$ is

$$
\left(\begin{array}{llll}
1 & 0 & 0 & 0 \\
1 & 1 & 0 & 0 \\
1 & 1 & 1 & 0 \\
1 & 0 & 1 & 1
\end{array}\right) .
$$

The last two rows stand for constructible representations.

3.6. If $|c|=5$ (so that $W$ is of type $E_{6}, E_{7}$, or $E_{8}$ ) we order $c$ using its bijection with $\left\{(1,1),(1, r),\left(g_{2}, 1\right),\left(g_{3}, 1\right),(1, \epsilon)\right\}$ in [L5, 4.11, 4.12, 4.13] (ordered from left to right); then $M_{c}$ is

$$
\left(\begin{array}{lllll}
1 & 0 & 0 & 0 & 0 \\
1 & 1 & 0 & 0 & 0 \\
1 & 1 & 1 & 0 & 0 \\
1 & 0 & 1 & 1 & 0 \\
1 & 2 & 0 & 0 & 1
\end{array}\right) .
$$

The last three rows stand for constructible representations.

3.7. If $|c|=11$ (so that $W$ is of type $F_{4}$ ) we write the elements of $c$ (notation of [L5, 4.10]) in the order

$$
12_{1}, 9_{3}, 6_{2}, 1_{3}, 16_{1}, 9_{2}, 4_{4}, 6_{1}, 4_{3}, 4_{1}, 1_{2}
$$


(from left to right); then $M_{c}$ is:

$$
\left(\begin{array}{lllllllllll}
1 & 0 & 0 & 0 & 0 & 0 & 0 & 0 & 0 & 0 & 0 \\
1 & 1 & 0 & 0 & 0 & 0 & 0 & 0 & 0 & 0 & 0 \\
1 & 1 & 1 & 0 & 0 & 0 & 0 & 0 & 0 & 0 & 0 \\
1 & 2 & 1 & 1 & 0 & 0 & 0 & 0 & 0 & 0 & 0 \\
1 & 1 & 1 & 0 & 1 & 0 & 0 & 0 & 0 & 0 & 0 \\
1 & 0 & 1 & 0 & 1 & 1 & 0 & 0 & 0 & 0 & 0 \\
1 & 2 & 1 & 1 & 1 & 0 & 1 & 0 & 0 & 0 & 0 \\
1 & 1 & 0 & 0 & 1 & 0 & 1 & 1 & 0 & 0 & 0 \\
1 & 0 & 0 & 0 & 1 & 1 & 0 & 1 & 1 & 0 & 0 \\
1 & 1 & 1 & 0 & 2 & 1 & 0 & 0 & 0 & 1 & 0 \\
1 & 0 & 1 & 0 & 1 & 2 & 0 & 0 & 1 & 0 & 1
\end{array}\right) .
$$

The last five rows stand for constructible representations.

3.8. If $|c|=17$ (so that $W$ is of type $E_{8}$ ) we write the elements of $c$ (with notation of [L5, 4.13.2] with subscripts omitted) in the order

4480, 5670, 4536, 1680, 1400, 70, 7168, 5600, 3150, 4200, 2688, 2016,

448, 1134, 1344, 420, 168

(from left to right); then $M_{c}$ is:

$$
\left(\begin{array}{lllllllllllllllll}
1 & 0 & 0 & 0 & 0 & 0 & 0 & 0 & 0 & 0 & 0 & 0 & 0 & 0 & 0 & 0 & 0 \\
1 & 1 & 0 & 0 & 0 & 0 & 0 & 0 & 0 & 0 & 0 & 0 & 0 & 0 & 0 & 0 & 0 \\
1 & 1 & 1 & 0 & 0 & 0 & 0 & 0 & 0 & 0 & 0 & 0 & 0 & 0 & 0 & 0 & 0 \\
1 & 2 & 1 & 1 & 0 & 0 & 0 & 0 & 0 & 0 & 0 & 0 & 0 & 0 & 0 & 0 & 0 \\
1 & 2 & 2 & 1 & 1 & 0 & 0 & 0 & 0 & 0 & 0 & 0 & 0 & 0 & 0 & 0 & 0 \\
1 & 3 & 3 & 3 & 2 & 1 & 0 & 0 & 0 & 0 & 0 & 0 & 0 & 0 & 0 & 0 & 0 \\
1 & 1 & 1 & 0 & 0 & 0 & 1 & 0 & 0 & 0 & 0 & 0 & 0 & 0 & 0 & 0 & 0 \\
1 & 2 & 2 & 1 & 1 & 0 & 1 & 1 & 0 & 0 & 0 & 0 & 0 & 0 & 0 & 0 & 0 \\
1 & 1 & 1 & 0 & 0 & 0 & 1 & 1 & 1 & 0 & 0 & 0 & 0 & 0 & 0 & 0 & 0 \\
1 & 1 & 1 & 0 & 1 & 0 & 1 & 1 & 0 & 1 & 0 & 0 & 0 & 0 & 0 & 0 & 0 \\
1 & 2 & 2 & 1 & 1 & 0 & 2 & 2 & 0 & 1 & 1 & 0 & 0 & 0 & 0 & 0 & 0 \\
1 & 1 & 1 & 0 & 0 & 0 & 2 & 1 & 1 & 1 & 1 & 1 & 0 & 0 & 0 & 0 & 0 \\
1 & 3 & 3 & 3 & 2 & 1 & 1 & 2 & 0 & 0 & 0 & 0 & 1 & 0 & 0 & 0 & 0 \\
1 & 2 & 1 & 1 & 0 & 0 & 1 & 2 & 1 & 0 & 0 & 0 & 1 & 1 & 0 & 0 & 0 \\
1 & 1 & 0 & 0 & 0 & 0 & 1 & 1 & 1 & 1 & 0 & 0 & 0 & 1 & 1 & 0 & 0 \\
1 & 0 & 0 & 0 & 0 & 0 & 1 & 0 & 1 & 1 & 0 & 1 & 0 & 0 & 1 & 1 & 0 \\
1 & 1 & 1 & 0 & 1 & 0 & 1 & 1 & 0 & 2 & 0 & 0 & 0 & 0 & 1 & 0 & 1
\end{array}\right) .
$$

The last seven rows stand for constructible representations.

3.9. For $N \geq 1$ let $S_{N}$ be the group of all permutations of $[1, N]$. If $a_{1} \geq a_{2} \geq \ldots$ is a partition of $N$ (written as $a_{1} a_{2} \ldots$ ) we say that a subgroup $H$ of $S_{N}$ is in $\mathcal{S}_{a_{1} a_{2} \ldots}$ if $H$ is conjugate to the subgroup of all permutations of $[1, N]$ which keep stable each of the subsets $\left[1, a_{1}\right],\left[a_{1}+1, a_{1}+a_{2}\right],\left[a_{1}+a_{2}+1, a_{1}+a_{2}+a_{3}\right], \ldots$. We say that a subgroup $H$ of $S_{N}$ (with $N \geq 4$ ) is in $\tilde{\mathcal{S}}_{N}$ if it is conjugate to the subgroup of all permutations of $[1, N]$ which act as an identity on $[1, N]-[1,4]$ and whose restriction to $[1,4]$ commutes with the permutation $1 \mapsto 4 \mapsto 1,2 \mapsto 3 \mapsto 2$.

The following results come from [L7].

If $|c|=1$ we have $\mathcal{G}_{c}=\{1\}$ and $\hat{\mathfrak{F}}_{c}$ consists of $\{1\}$. 
In the setup of 3.3 or 3.4 we have $\mathcal{G}_{c}=S_{2}$ and $\hat{\mathfrak{F}}_{c}$ consists of $S_{2},\{1\}$.

In the setup of 3.5 or 3.6 we have $\mathcal{G}_{c}=S_{3}$ and $\hat{\mathfrak{F}}_{c}$ consists of $S_{3},\{1\}$ and the subgroups of $S_{3}$ in $\mathcal{S}_{21}$.

In the setup of 3.7 we have $\mathcal{G}_{c}=S_{4}$ and $\hat{\mathfrak{F}}_{c}$ consists of $S_{4},\{1\}$ and the subgroups of $S_{4}$ in $\mathcal{S}_{31}, \mathcal{S}_{22}, \mathcal{S}_{211}, \tilde{\mathcal{S}}_{4}$.

In the setup of 3.8 we have $\mathcal{G}_{c}=S_{5}$ and $\hat{\mathfrak{F}}_{c}$ consists of $S_{5},\{1\}$ and the subgroups of $S_{5}$ in $\mathcal{S}_{41}, \mathcal{S}_{32}, \mathcal{S}_{311}, \mathcal{S}_{221}, \mathcal{S}_{2111}, \tilde{\mathcal{S}}_{5}$.

3.10. We describe the set $\tilde{\Theta}_{c}$ in each of the cases 3.23 .8 ,

If $|c|=1, \tilde{\Theta}_{c}$ consists of $(1,1)$. (We shall write 1 instead of $\{1\}$.)

In the setup of 3.3 or $3.4, \tilde{\Theta}_{c}$ consists of $\left(1, S_{2}\right),\left(S_{2}, S_{2}\right),(1,1)$.

In the setup of 3.5 or 3.6. $\tilde{\Theta}_{c}$ consists of $\left(1, S_{3}\right),\left(1, H_{21}\right),\left(H_{21}, H_{21}\right),\left(S_{3}, S_{3}\right),(1,1)$ where $H_{21}$ runs through $\mathcal{S}_{21}$.

In the setup of 3.7, $\tilde{\Theta}_{c}$ consists of

$$
\begin{aligned}
& \left(1, S_{4}\right),\left(1, H_{31}\right),\left(1, H_{22}\right),\left(1, H_{211}\right),\left(\tilde{H}_{211}, H_{22}\right),\left(\tilde{H}_{22}, \tilde{H}\right), \\
& \left(H_{211}, H_{211}\right),\left(H_{31}, H_{31}\right),\left(S_{4}, S_{4}\right),\left(H_{22}, H_{22}\right),(\tilde{H}, \tilde{H}),(1,1),(1, \tilde{H}),
\end{aligned}
$$

where $H_{211}$ runs through $\mathcal{S}_{211}, H_{31}$ runs through $\mathcal{S}_{31}, H_{22}$ runs through $\mathcal{S}_{22}, \tilde{H}$ runs through $\tilde{\mathcal{S}}_{4}$; for $H_{22} \in \mathcal{S}_{22}, \tilde{H}_{211}$ denotes one of the two subgroups in $\mathcal{S}_{211}$ contained in $H_{22}$; for $\tilde{H} \in \tilde{\mathcal{S}}_{4}, \tilde{H}_{22}$ denotes the unique subgroup in $\mathcal{S}_{22}$ contained in $\tilde{H}$.

In the setup of 3.8 , $\tilde{\Theta}_{c}$ consists of

$$
\begin{aligned}
& \left(1, S_{5}\right),\left(1, H_{41}\right),\left(1, H_{32}\right),\left(1, H_{311}\right),\left(1, H_{221}\right),\left(1, H_{2111}\right),\left(\tilde{H}_{2111}, H_{32}\right), \\
& \left(\tilde{H}_{2111}, H_{221}\right),\left(\tilde{H}_{311}, H_{32}\right),\left(\tilde{H}_{221}, \tilde{H}\right),\left(H_{221}, H_{221}\right),\left(H_{32}, H_{32}\right), \\
& \left(H_{2111}, H_{2111}\right),\left(H_{311}, H_{311}\right),\left(H_{41}, H_{41}\right),\left(S_{5}, S_{5}\right),(\tilde{H}, \tilde{H}),(1,1),(1, \tilde{H}),
\end{aligned}
$$

where $H_{2111}$ runs through $\mathcal{S}_{2111}, H_{221}$ runs through $\mathcal{S}_{221}, H_{32}$ runs through $\mathcal{S}_{32}$, $H_{311}$ runs through $\mathcal{S}_{311}, H_{41}$ runs through $\mathcal{S}_{41}, \tilde{H}$ runs through $\tilde{\mathcal{S}}_{5}$; for $H_{221} \in \mathcal{S}_{221}$, $\tilde{H}_{2111}$ denotes one of the two subgroups in $\mathcal{S}_{2111}$ contained in $H_{221}$; for $H_{32} \in \mathcal{S}_{32}$, $\tilde{H}_{2111}$ denotes the unique subgroup in $\mathcal{S}_{2111}$ which is a normal subgroup of $H_{32}$ and $\tilde{H}_{311}$ denotes the unique subgroup in $\mathcal{S}_{311}$ which is a normal subgroup of $H_{32}$; for $\tilde{H} \in \tilde{\mathcal{S}}_{5}, \tilde{H}_{221}$ denotes the unique subgroup in $\mathcal{S}_{221}$ contained in $\tilde{H}$.

3.11. We define the set $\Theta_{c}$ in each of the cases $3.2\left[3.8\right.$ by removing from $\tilde{\Theta}_{c}$ the pair $(1,1)$ whenever $c$ is anomalous (see 0.3) and by removing the pairs $(1, \tilde{H})$ with $\tilde{H}$ in $\tilde{\mathcal{S}}_{4}$ or $\tilde{\mathcal{S}}_{5}$ whenever $\tilde{\mathcal{S}}_{4}$ or $\tilde{\mathcal{S}}_{5}$ is defined. This guarantees that for $\left(H, H^{\prime}\right) \in \Theta_{c}$, $H^{\prime} / H$ is isomorphic to a product of symmetric groups.

If $|c|=1, \Theta_{c}$ consists of $(1,1)$.

In the setup of 3.3. $\Theta_{c}$ consists of $\left(1, S_{2}\right),\left(S_{2}, S_{2}\right)$.

In the setup of 3.4. $\Theta_{c}=\tilde{\Theta}_{c}$ consists of $\left(1, S_{2}\right),\left(S_{2}, S_{2}\right),(1,1)$.

In the setup of 3.5, $\Theta_{c}$ consists of $\left(1, S_{3}\right),\left(1, H_{21}\right),\left(H_{21}, H_{21}\right),\left(S_{3}, S_{3}\right)$ (notation of 3.10 . 
In the setup of 3.6, $\Theta_{c}=\tilde{\Theta}_{c}$ consists of $\left(1, S_{3}\right),\left(1, H_{21}\right),\left(H_{21}, H_{21}\right),\left(S_{3}, S_{3}\right),(1,1)$ (notation of 3.10 ).

In the setup of 3.7. $\Theta_{c}$ consists of

$$
\begin{aligned}
& \left(1, S_{4}\right),\left(1, H_{31}\right),\left(1, H_{22}\right),\left(1, H_{211}\right),\left(\tilde{H}_{211}, H_{22}\right),\left(\tilde{H}_{22}, \tilde{H}\right), \\
& \left(H_{211}, H_{211}\right),\left(H_{31}, H_{31}\right),\left(S_{4}, S_{4}\right),\left(H_{22}, H_{22}\right),(\tilde{H}, \tilde{H}),
\end{aligned}
$$

(notation of 3.10).

In the setup of 3.8 , $\Theta_{c}$ consists of

$$
\begin{aligned}
& \left(1, S_{5}\right),\left(1, H_{41}\right),\left(1, H_{32}\right),\left(1, H_{311}\right),\left(1, H_{221}\right),\left(1, H_{2111}\right),\left(\tilde{H}_{2111}, H_{32}\right), \\
& \left(\tilde{H}_{2111}, H_{221}\right),\left(\tilde{H}_{311}, H_{32}\right),\left(\tilde{H}_{221}, \tilde{H}\right),\left(H_{221}, H_{221}\right),\left(H_{32}, H_{32}\right), \\
& \left(H_{2111}, H_{2111}\right),\left(H_{311}, H_{311}\right),\left(H_{41}, H_{41}\right),\left(S_{5}, S_{5}\right),(\tilde{H}, \tilde{H}),
\end{aligned}
$$

(notation of 3.10).

In each case, the number of $\mathcal{G}_{c}$-orbits on $\Theta_{c}$ is equal to $|c|$. By computation we see that $S_{H, H^{\prime}}$ with $\left(H, H^{\prime}\right)$ running through a set of representatives for the $\mathcal{G}_{c^{-}}$-orbits on $\Theta_{c}$ are of the form $E_{H, H^{\prime}}$ (see 0.3 ) where $E_{H, H^{\prime}} \in \mathcal{R}_{c}$ runs through the

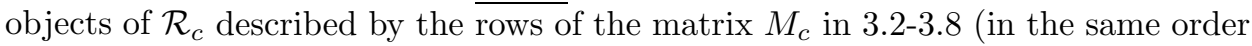
as the one used in the description of $\Theta_{c}$ given above). These objects form a basis of $\mathcal{G}_{c}$, due to the form of the matrix $M_{c}$. Now Theorem 0.4 follows in our case.

\section{Proof of Theorem 0.7}

4.1. Let $H \subset H^{\prime}$ be subgroups of the finite group $\Gamma$ with $H$ normal in $H^{\prime}$. For any $x \in \Gamma$ we consider the set $S(x)$ of all $\mu$ in $\Gamma / H^{\prime}$ such that for some $\gamma$ in $\Gamma / H$ contained in $\mu$ we have $x \gamma=\gamma$. Now $Z(x)$ acts on $S(x)$ by $y: \mu \mapsto y \mu$. For any $(x, \sigma) \in M(\Gamma)$ let $N_{x, \sigma} \in \mathbf{N}$ be the multiplicity of $\sigma$ in the permutation representation of $Z(x)$ on $S(x)$. We have

$$
N_{x, \sigma}=|Z(x)|^{-1} \sum_{y \in Z(x)} \sharp(\mu \in S(x) ; y \mu=\mu) \operatorname{tr}(y, \sigma),
$$

where

$$
\sharp(\mu \in S(x) ; y \mu=\mu)
$$$$
=\sharp\left(\mu \in \Gamma / H^{\prime} \text {; for some } u \in \Gamma \text { we have } x u H=u H, \mu=u H^{\prime}, y u H^{\prime}=u H^{\prime}\right) \text {. }
$$

If the previous three equations hold for some $u$, then they hold for $u h^{\prime}$ for any $h^{\prime} \in$ $H^{\prime}$. (Indeed, $x u h^{\prime} H=u h^{\prime} H$ since $h^{\prime} H=H h^{\prime}$, and $\mu=u h^{\prime} H^{\prime}, y u h^{\prime} H^{\prime}=u h^{\prime} H^{\prime}$.) Thus,

$$
\sharp(\mu \in S(x) ; y \mu=\mu)=\sharp\left(u \in \Gamma ; x u H=u H, y u H^{\prime}=u H^{\prime}\right) /\left|H^{\prime}\right|
$$

and

$$
\begin{aligned}
& N_{x, \sigma}=|Z(x)|^{-1}\left|H^{\prime}\right|^{-1} \sum_{y \in Z(x)} \sharp\left(u \in \Gamma ; x u H=u H, y u H^{\prime}=u H^{\prime}\right) \operatorname{tr}(y, \sigma) \\
& =|Z(x)|^{-1}\left|H^{\prime}\right|^{-1} \sum_{y \in Z(x), u \in \Gamma ; x u H=u H, y u H^{\prime}=u H^{\prime}} \operatorname{tr}(y, \sigma) .
\end{aligned}
$$


Let $f=\sum_{(x, \sigma) \in M(\Gamma)} N_{x, \sigma}(x, \sigma) \in \mathbf{C}[M(\Gamma)]$. We have $f=S_{H, H^{\prime}}$. We write $A(f)=\sum_{\left(x^{\prime}, \sigma^{\prime}\right) \in M(\Gamma)} N_{x^{\prime}, \sigma^{\prime}}^{\prime}\left(x^{\prime}, \sigma^{\prime}\right)$ with $N_{x^{\prime}, \sigma^{\prime}}^{\prime} \in \mathbf{C}$. We have

$$
\begin{aligned}
& N_{x^{\prime}, \sigma^{\prime}}^{\prime}=\sum_{(x, \sigma) \in M(\Gamma)} N_{x, \sigma}(x, \sigma),\left(x^{\prime}, \sigma^{\prime}\right) \\
& =\sum_{(x, \sigma) \in M(\Gamma)}|Z(x)|^{-1}\left|H^{\prime}\right|^{-1}|Z(x)|^{-1}\left|Z\left(x^{\prime}\right)\right|^{-1} \sum_{y \in Z(x), u \in \Gamma ; x u H=u H, y u H^{\prime}=u H^{\prime}} \\
& \quad \sum_{z \in \Gamma ; z x z^{-1} x^{\prime}=x^{\prime} z x z^{-1}} \frac{\operatorname{tr}\left(z x z^{-1}, \sigma^{\prime}\right)}{\operatorname{tr}}\left(z^{-1} x^{\prime} z, \sigma\right) \operatorname{tr}(y, \sigma) \\
& =\sum_{x \in \Gamma}|\Gamma|^{-1}\left|H^{\prime}\right|^{-1}|Z(x)|^{-1}\left|Z\left(x^{\prime}\right)\right|^{-1} \sum_{y \in Z(x), u \in \Gamma ; x u H=u H, y u H^{\prime}=u H^{\prime}} \\
& \quad \sum_{z \in \Gamma ; z x z^{-1} x^{\prime}=x^{\prime} z x z^{-1}} \frac{\operatorname{tr}\left(z x z^{-1}, \sigma^{\prime}\right)}{\sum_{\sigma \in \operatorname{Irr}(Z(x)} \operatorname{tr}\left(z^{-1} x^{\prime} z, \sigma\right) \operatorname{tr}(y, \sigma) .}
\end{aligned}
$$

The last sum over $\sigma$ equals $|Z(x) \cap Z(y)|$ if $z^{-1} x^{\prime} z=a y^{-1} a^{-1}$ for some $a \in Z(x)$ and equals 0 otherwise. Hence

$$
\begin{gathered}
N_{x^{\prime}, \sigma^{\prime}}^{\prime}=\sum_{x \in \Gamma}|\Gamma|^{-1}\left|H^{\prime}\right|^{-1}|Z(x)|^{-1}\left|Z\left(x^{\prime}\right)\right|^{-1} \\
\sum_{z \in \Gamma ; z x z^{-1} x^{\prime}=x^{\prime} z x z^{-1}, a^{-1} n Z(x), z^{-1} x^{\prime} z=a y^{-1} a^{-1}} \sum_{y \in Z(x), u \in \Gamma ; x u H=u H, y u H^{\prime}=u H^{\prime}} \frac{\operatorname{tr}\left(z x z^{-1}, \sigma^{\prime}\right) .}{}
\end{gathered}
$$

We substitute $z_{1}=z a$. We get

$$
\begin{gathered}
N_{x^{\prime}, \sigma^{\prime}}^{\prime}=\sum_{x \in \Gamma}|\Gamma|^{-1}\left|H^{\prime}\right|^{-1}|Z(x)|^{-1}\left|Z\left(x^{\prime}\right)\right|^{-1} \\
\sum_{z_{1} \in \Gamma ; z_{1} x z_{1}^{-1} x^{\prime}=x^{\prime} z_{1} x z_{1}^{-1}, a^{-1} n Z(x), z_{1}^{-1} x^{\prime} z_{1}=y^{-1}} \sum_{\frac{y \in Z(x), u \in \Gamma ; x u H=u H, y u H^{\prime}=u H^{\prime}}{\operatorname{tr}\left(z_{1} x z_{1}^{-1}, \sigma^{\prime}\right) .}}
\end{gathered}
$$

We can eliminate $a$ and change $z_{1}$ to $z$. We get

$$
\begin{gathered}
N_{x^{\prime}, \sigma^{\prime}}^{\prime}=\sum_{x \in \Gamma}|\Gamma|^{-1}\left|H^{\prime}\right|^{-1}\left|Z\left(x^{\prime}\right)\right|^{-1} \sum_{y \in Z(x), u \in \Gamma ; x u H=u H, y u H^{\prime}=u H^{\prime}} \\
\sum_{z \in \Gamma ; z x z^{-1} x^{\prime}=x^{\prime} z x z^{-1}, z^{-1} x^{\prime} z=y^{-1}} \frac{\sum_{\operatorname{tr}\left(z x z^{-1}, \sigma^{\prime}\right) .}}{}
\end{gathered}
$$

We substitute $x_{1}=u^{-1} x u, y_{1}=u^{-1} y u, z_{1}=z u$. We get

$$
\begin{aligned}
& N_{x^{\prime}, \sigma^{\prime}}^{\prime}=\sum_{x_{1} \in \Gamma}|\Gamma|^{-1}\left|H^{\prime}\right|^{-1}\left|Z\left(x^{\prime}\right)\right|^{-1} \sum_{y_{1} \in Z\left(x_{1}\right), u \in \Gamma ; x_{1} H=H, y_{1} H^{\prime}=H^{\prime}} \\
& \sum_{z_{1} \in \Gamma ; z_{1} x_{1} z_{1}^{-1} x^{\prime}=x^{\prime} z_{1} x_{1} z_{1}^{-1}, z_{1}^{-1} x^{\prime} z_{1}=y_{1}^{-1}} \overline{\operatorname{tr}\left(z_{1} x_{1} z_{1}^{-1}, \sigma^{\prime}\right)} .
\end{aligned}
$$

We can eliminate $u$ and change $x_{1}, y_{1}, z_{1}$ to $x, y, z$. We get

$$
\begin{aligned}
& N_{x^{\prime}, \sigma^{\prime}}^{\prime}=\left|H^{\prime}\right|^{-1}\left|Z\left(x^{\prime}\right)\right|^{-1} \sum_{x \in H, y \in Z(x) \cap H^{\prime}} \frac{\sum_{\operatorname{tr}\left(z x z^{-1}, \sigma^{\prime}\right)}}{\operatorname{tr} z x z^{-1} x^{\prime}=x^{\prime} z x z^{-1}, z^{-1} x^{\prime} z=y^{-1}}
\end{aligned}
$$


Here the condition $z x z^{-1} x^{\prime}=x^{\prime} z x z^{-1}$ follows from $z^{-1} x^{\prime} z=y^{-1}, y x=x y$. Hence

$$
N_{x^{\prime}, \sigma^{\prime}}^{\prime}=\left|H^{\prime}\right|^{-1}\left|Z\left(x^{\prime}\right)\right|^{-1} \sum_{x \in H, y \in Z(x) \cap H^{\prime}} \sum_{z \in \Gamma ; z^{-1} x^{\prime} z=y^{-1}} \overline{\operatorname{tr}\left(z x z^{-1}, \sigma^{\prime}\right)},
$$

that is,

$$
N_{x^{\prime}, \sigma^{\prime}}^{\prime}=\left|H^{\prime}\right|^{-1}\left|Z\left(x^{\prime}\right)\right|^{-1} \sum_{x \in H} \sum_{z \in \Gamma ; z^{-1}} \overline{x^{\prime} z \in Z(x) \cap H^{\prime}} \overline{\operatorname{tr}\left(z x z^{-1}, \sigma^{\prime}\right)} .
$$

We substitute $z x z^{-1}=x_{1}$. We get

$$
N_{x^{\prime}, \sigma^{\prime}}^{\prime}=\left|H^{\prime}\right|^{-1}\left|Z\left(x^{\prime}\right)\right|^{-1} \sum_{x_{1} \in \Gamma, z \in \Gamma ; x^{\prime} \in Z\left(x_{1}\right) \cap z H^{\prime} z^{-1}, x_{1} \in z H z^{-1}} \overline{\operatorname{tr}\left(x_{1}, \sigma^{\prime}\right)},
$$

that is,

$$
\sum_{z \in \Gamma ; z^{-1} x^{\prime} z \in H^{\prime}}^{N_{x^{\prime}, \sigma^{\prime}}^{\prime}} \sum_{x_{1} \in Z\left(x^{\prime}\right) \cap z H z^{-1}} \overline{\operatorname{tr}\left(x_{1}, \sigma^{\prime}\right)}
$$

and

$$
\begin{aligned}
& N_{x^{\prime}, \sigma^{\prime}}^{\prime}=\left|H^{\prime}\right|^{-1}\left|Z\left(x^{\prime}\right)\right|^{-1} \\
& \sum_{z \in \Gamma ; z^{-1} x^{\prime} z \in H^{\prime}}\left(1: \sigma^{\prime} \mid\left(Z\left(x^{\prime}\right) \cap z H z^{-1}\right)\right)\left|Z\left(x^{\prime}\right) \cap z H z^{-1}\right|,
\end{aligned}
$$

where : denotes multiplicity. Thus we have

$$
N_{x^{\prime}, \sigma^{\prime}} \in \mathbf{Q}_{\geq 0}
$$

so that $A(f) \in M(\Gamma)_{\geq 0}$. Since $f \in M(\Gamma)_{\geq 0}$ is obvious we see that $f$ is bipositive.

This proves Theorem 0.7 .

\section{RefERENCES}

[L1] G. Lusztig, A class of irreducible representations of a Weyl group, Nederl. Akad. Wetensch. Indag. Math. 41 (1979), no. 3, 323-335. MR546372

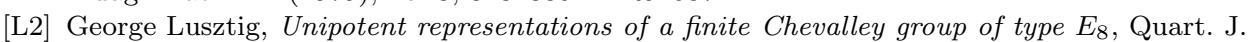
Math. Oxford Ser. (2) 30 (1979), no. 119, 315-338, DOI 10.1093/qmath/30.3.315. MR.545068

[L3] George Lusztig, Unipotent characters of the symplectic and odd orthogonal groups over a finite field, Invent. Math. 64 (1981), no. 2, 263-296, DOI 10.1007/BF01389170. MR629472

[L4] George Lusztig, A class of irreducible representations of a Weyl group. II, Nederl. Akad. Wetensch. Indag. Math. 44 (1982), no. 2, 219-226. MR662657

[L5] George Lusztig, Characters of reductive groups over a finite field, Annals of Mathematics Studies, vol. 107, Princeton University Press, Princeton, NJ, 1984 . MR742472

[L6] George Lusztig, Sur les cellules gauches des groupes de Weyl (French, with English summary), C. R. Acad. Sci. Paris Sér. I Math. 302 (1986), no. 1, 5-8. MR827096

[L7] G. Lusztig, Leading coefficients of character values of Hecke algebras, The Arcata Conference on Representations of Finite Groups (Arcata, Calif., 1986), Proc. Sympos. Pure Math., vol. 47, Amer. Math. Soc., Providence, RI, 1987, pp. 235-262. MR933415

Department of Mathematics, Room 2-365, Massachusetts Institute of Technology, Cambridge, Massachusetts 02139

Email address: gyuri@mit.edu 